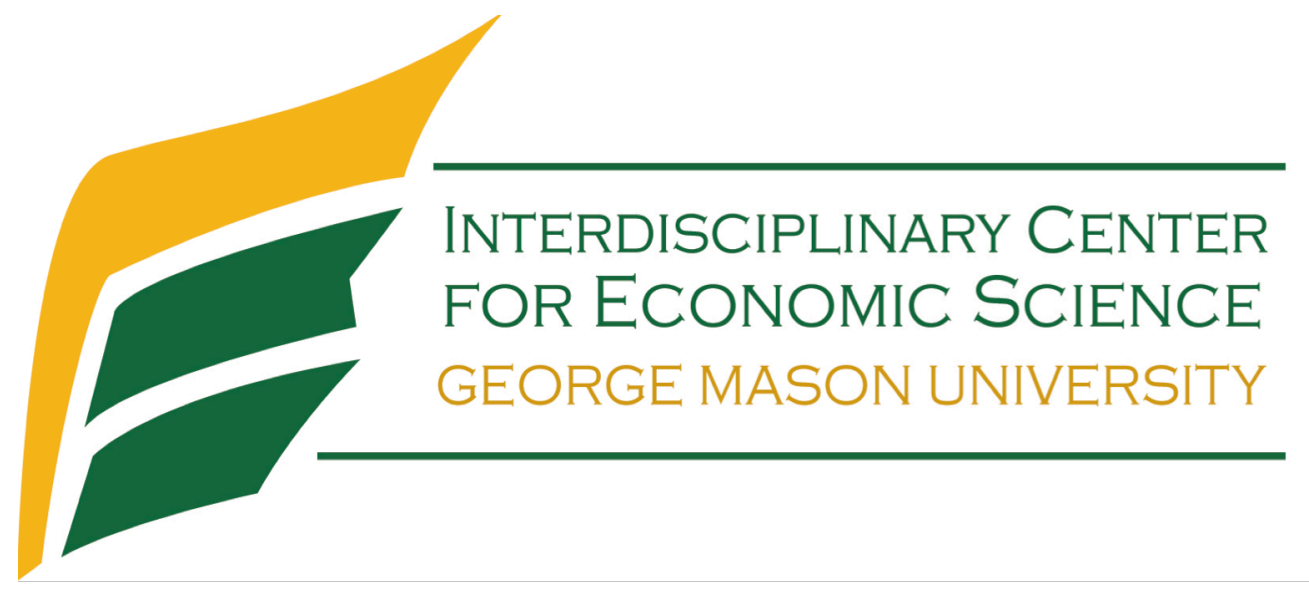

\title{
Demanding or Deferring? The Economic Value of Communication with Attitude
}

Siyu Wang and Daniel Houser

July 2015

Discussion Paper

Interdisciplinary Center for Economic Science

4400 University Drive, MSN 1B2, Fairfax, VA 22030

Tel: +1-703-993-4719 Fax: +1-703-993-4851

ICES Website: $h$ ttp://ices.gmu.edu

ICES RePEc Archive Online at: http://edirc.repec.org/data/icgmuus.html 


\title{
Demanding or Deferring? The Economic Value of Communication with Attitude
}

\author{
Siyu Wang ${ }^{* \dagger}$ and Daniel Houser*
}

July 2015

\begin{abstract}
This paper investigates why cheap-talk natural language communication is systematically found to promote coordination better than predetermined intention signaling. We hypothesize the reason is that, when communicating with natural language, people both use and respond to intentions and attitudes, where attitude indicates the strength of a message sender's desire to have her message followed. We test our hypothesis using controlled laboratory experiments in both the United States and China. We find (i) free-form messages do include both signaled intentions and attitudes; (ii) people respond both to intentions and attitudes when making decisions; and (iii) the use of attitude significantly improves coordination. Moreover, while males and females recognize and respond to intentions and attitudes equally well, we find females are more likely to send more demanding signals than males, while males send messages focused more on the equilibrium outcome than attitude.
\end{abstract}

Keywords communication; coordination; experiment; attitude; gender

*Interdisciplinary Center for Economic Science, Department of Economics, George Mason University, 4400 University Blvd. MSN 1B2, Fairfax, VA. 22030. S.W. and D.H. equally contributed to this work.

${ }^{\dagger}$ To whom correspondence should be addressed. Email: swang14@gmu.edu 


\section{INTRODUCTION}

Language is a powerful and complex human tool facilitating social and economic decisions. Over the last decades there has been significant theoretical and empirical progress towards understanding how communication improves coordination. While much of this early progress has occurred within the context of one-dimensional intention signaling models, natural language communication has a more richly detailed structure that can convey multiple meanings concisely. That this structure has evidently survived an evolutionary process has led some economists to argue for its importance not only to understanding grammar (e.g. Selten and Warglien 2007) but also for understanding human social and economic decisions (e.g. Rubinstein 2000). Consequently, it has been long-argued that the existence of a rich language should play a more prominent role in game theory (see Farrell, 1993). At the same time, others have demonstrated empirically that, in relation to constrained signaling, efficient economic outcomes emerge more readily when players can communicate using rich natural language (see Charness and Dufwenberg 2006, 2010; Cooper and Kühn, 2014 and 2015). Our aim here is to present and test a formal framework that predicts people both use and respond to multi-meaning natural language in a way that improves coordination. In doing so we bridge theory (Farrell 1993, Rabin 1994) and experiment (Charness and Dufwenberg 2006, 2010; Cooper and Kühn, 2014 and 2015) in a way that sheds light on why and how rich, multi-meaning language promotes efficient economic outcomes.

To fix ideas, consider routine discussions over where to dine or which film to view. If everyone conveys indifference, by stating that it's "up to you", or "I do not care", the conversation is likely to be long and inefficient. At the same time, the same is true if all report a preferred option without a clear attitude. For example, one person says, "I feel like French," and another responds, "I'm thinking Indian," a different long conversation ensues. People typically solve these problems by making statements that combine intention signaling with attitude ${ }^{1}$, that is, by indicating strength of desire to have one's suggestion followed. For example, one person's, "I really want French tonight" meeting another's "I'm thinking Indian, but it's up to you" will lead to immediate agreement. Intuitively, it makes good sense to communicate in a way that allows common ground to be rapidly found. Our model shows this can be done when

\footnotetext{
${ }^{1}$ In the psychology literature, attitude is understood as one component of a broader model of behavior, which includes social norms and behavioral intentions. The way define attitude in this paper is closely related to the behavioral intentions part of the concept. (see Osgood et al. 1957, Fishbein 1967c, Fishbein 1975).
} 
communication includes multiple independent meanings, and in particular when requests are made with attitude.

We capture this intuition by analyzing two-way communication with two-dimensional meanings pre-played before coordination game. In our model, players play a two-stage game in which they simultaneously send each other cheap-talk messages in the first stage and simultaneously take actions in the second stage. We assume the language space players use to communicate is two-dimensional, common and complete. That is, players understand each other's messages, each of which includes a signaled equilibrium (so-called E-meaning; Rabin 1991, 1994) as well as an attitude (which we denote as A-meaning). We model an environment in which there are only two possible pure-strategy equilibria and two possible attitudes. Thus, the E-meaning indicates which of the two possible pure-strategy equilibria the player intends to play, while the A-meaning indicates whether the player has either a weak or strong desire to have her message followed.

In a cheap-talk environment, it is well known that standard refinements of perfect Bayesian equilibria do not eliminate equilibria ${ }^{2}$. To eliminate unreasonable equilibria, Farrell (1993) introduced Credibility, the intuition for which is that communication works well when there is no incentive to lie. Demichelis and Weibull (2008) include lexicographic preference for honesty, second to material preferences, to capture a similar idea. We apply the notion of Credibility in a communication environment with multi-dimensional meanings to define an agreementequilibrium, which requires that players act in accordance with the E-meaning of their messages when the action is a best response to the E-meaning of her partner's message. Furthermore, we show that taking advantage of A-meaning, which reveals one's desire to have her message followed, can eliminate bad equilibria in a symmetric coordination game.

In particular, we introduce Attitude Ranking, which posits that players indicating weaker desire to have her message followed (that is, those who are more willing to defer) cede decision authority to the player indicating stronger desire (that is, those who are more demanding). With this condition, we prove the existence of another group of equilibria, denoted negotiatedequilibria, which require that players act in accordance with the relatively more demanding player's E-meaning when paired players' messages differ in both E-meaning and A-meaning. We

\footnotetext{
${ }^{2}$ See Crawford (1998) for brief summary of the literature.
} 
show that negotiated-equilibria, in which one player concedes to the other players' requested equilibrium can happen in one-shot simultaneous communication.

In our simple example with only two E-meanings and two A-meanings, communication failure can occur whenever players indicate differing E-meanings along with the same Ameaning. In these cases players will play as though communication did not occur. It is easy to see, however, that if the A-meaning is continuous then communication failure occurs with probability zero. The intuition is that in this case A-meanings differ with probability one, meaning they can surely be used to arbitrate disagreement over E-meanings. To the extent that time is costly, because communicating with attitude allows more rapid agreement it is also in this sense efficient. In our experiments below, we are able to test not only whether people naturally and without prompting communicate with attitude, as well as whether attitude is more finelygrained than the two-dimensions we consider in our model.

We test whether people use and respond to attitude in the way theory suggests by using laboratory implementations of complete-information pure coordination games with pre-play communication. Consistent with our model's predictions, we find (i) natural language messages are made with attitude; (ii) people consider both their partner's E-meaning and A-meaning when making their decisions; and (iii) the use of attitude improves coordination by enabling negotiated equilibria to emerge in one-shot simultaneous communication.

We compare our results using natural language to games where the nature of communication must follow a predetermined structure. We consider structured communication in which only an E-meaning can be conveyed, as well as treatments where both an E-meaning and one of two $A$ meanings can be conveyed. We find that free-form communication promotes coordination better than occurs in either of these cases. Our analysis also reveals gender effects. Although males and females recognize and respond to attitude equally well, they create different types of messages. We find females are more likely to use stronger (more demanding) recommendations than males, and that males generally focus more on which equilibrium request to send rather than which attitude to use.

Economic theory has long conjectured that natural language communication facilitates efficient economic outcomes because it includes a rich, multi-meaning structure that people use and to which people respond (e.g. Marschack, 1965). To our knowledge, our paper is the first direct test of this conjecture, and our findings lend support to the related body of theory. More 
generally, our results suggest that people communicate with attitude because it is efficient: doing so allows agreement to be reached more rapidly than might otherwise be possible.

The remainder of this paper is organized as follows. Section 2 reviews the literature on preplay communication. Section 3 models communication with two-dimensional meanings. Section 4 describes the design of our experimental test. Section 5 summarizes and discusses our main findings. Section 6 offers concluding remarks and further discussion.

\section{LITERATURE REVIEW}

\subsection{Intention Signaling}

The modern theory of cheap-talk originates with Aumann (1974), who introduced "cheaptalk" communication where agents engage in costless, non-binding, non-verifiable, pre-play talk before choosing their moves. Aumann argues that players will reach some agreement, and that since no external enforcement is available they can only consider self-enforcing Nash outcomes. Farrell and Maskin (1987) confirms that cheap-talk can generate a selection of self-enforcing agreements. Aumann (1990) adds subtlety to this result by noting that the effectiveness of communication in promoting coordination depends crucially on the payoff structure of the game (see also Farrell and Rabin, 1996).

Farrell $(1987,1988)$ took the important step of studying restricted intention signaling in simple sequential games with complete information, where an underlying game is preceded by one or more rounds of structured pre-play communication in which players make nonbinding announcements about their intended moves. (Farrell (1987) studies Battle of the Sexes and Farrell (1988) considers a finite n-person game.) Farrell suggested cheap-talk need not be ignored, and moreover it could make equilibrium focal.

Rabin $(1991,1994)$ extended the analyses of Farrell $(1987,1988)$. In Rabin's model, players make repeated, simultaneous statements about their intended moves before they play a coordination game. He made it possible to combine the assumptions that players maximize expected payoffs given beliefs with a variety of behaviorally motivated restrictions on beliefs. He generalized Farrell's (1987) result to an unlimited number of rounds of communication.

Several studies have looked at the strategic aspects of cheap talk. Crawford (2003) allows

for the possibility of bounded strategic rationality and rational players' responses to the misrepresentation of intentions. Demichelis and Weibull (2008) generalized cheap-talk models 
by introducing a small cost of lying, and find this so-called lexicographic communication game is evolutionarily stable if and only if it results in a unique Pareto-efficient outcome. Ellingsen and Östling (2010) used a level-k model of strategic thinking to describe players' beliefs in games with one-way and two-way communications of intentions. They found that communication facilitates coordination in common interest games with positive spillovers and strategic complementarities.

Experimental evidences suggest that the effectiveness of cheap-talk in promoting coordination depends crucially on the payoff structure of the underlying game, but also the structure of communication mechanisms (e.g. Cooper et al. 1989, 1992; Holt and Davis 1990; Van Huyck et al. 1993; Charness 2000; Clark et al. 2001; Charnes and Grosskopf, 2004; Duffy and Feltovich 2002, 2006, Blume and Ortman 2007). For instance, Clark et al. (2001) tested Aumann's conjecture with two-way signals and showed, consistent with theory, informative communication does not necessarily lead to Pareto-efficient equilibrium outcomes. Cooper et al. (1989) found one-way communication resolves coordination problems in the Battle of Sexes game, while two-way simultaneous communication is of less value in solving coordination problems. Cooper et al. (1992) confirmed that two-way communication does not always decrease the frequency of coordination failure in different types of coordination games.

Blume and Ortmann (2007) studied Minimum and Median games ${ }^{3}$ where multiple Paretoranked equilibria exist. In their design, all (nine) players send numerical messages simultaneously to indicate which of the (seven) options they intend to choose. Cheap talk facilitates coordination in games with a unique efficient equilibrium. Choi and Lee (2014) studied the role of network structure of pre-play communication in determination of outcomes in coordination games. In addition to providing evidence that increasing the length of communication both improves the chance of successful coordination and reduces the asymmetry in the distribution of outcomes, they also discovered substantial variations in both efficiency and equity of coordination across networks.

\subsection{Natural Language Communication}

\footnotetext{
${ }^{3}$ Van Huyck, Battalio and Beil (1990) designed the so-called Minimum and Median games, where coordination among multiple players are required for efficient equilibrium. They showed that adding a pre-play auction enables players to coordinate on Pareto-efficient equilibrium.
} 
Formal game theoretic modeling of free-form communication is rather limited. An influential theoretical advance closely related was provided by Farrell (1993), where he carefully discusses the meaning of a message. He argues that a message may have meaning in one of three ways. First, a meaning may be established by use: Wittgenstein (1957) urged "Don't ask the meaning; ask the use." The meaning of messages that are used in equilibrium, for example, is established by Bayes's rule, which tells us their meaning-in-use. Second, messages may have meanings that can be determined, or at least somewhat restricted, by introspection. This yields restrictions on out-of-equilibrium beliefs in generic signaling games; but not cheap-talk games. Finally - and this is the key element absent in previous analyses - a message may have a focal meaning, especially if it is phrased in a preexisting language. This is the first time, to the best of our knowledge, that game theorist argues that the existence of a rich, common language among agents can promote meaningful communication. It will become clear below that our model builds closely on this previous research by Farrell and Rabin.

In the laboratory, free-form cheap-talk has been shown to improve efficiency significantly in games including prisoners' dilemmas (Dawes, MacTavish, and Shaklee 1977), sealed bid auctions (Isaac and Walker 1988), as well as games of signaling (Cooper and Kagel 2005) and coordination (Brandts and Cooper 2007). Many studies implement pre-play cheap-talk as freeform written messages, including paper-pencil experiment as well as chat-box (e.g. Cooper and Kagel 2005; Xiao and Houser 2005, 2009, 2011; Charness and Dufwenberg 2006; Brandts and Cooper 2007; Schotter and Sopher 2007; Kimbrough et al. 2008; Ellingsen and Johannesson 2007; Sutter and Strassmair 2009; Heinnig Schmidt et al. 2008; Lundquist et al. 2009, Cason et al. 2012). Some studies also use face-to-face communication (Isaac, Ramey and Williams 1984; Daughety and Forsythe 1987a, 1987b; Binger et al. 1990; Valley et al. 1998).

More recently, a few experimental papers have suggested that free-form communication works better than restricted-form in facilitating efficient outcomes. Charness and Dufwenberg (2006) studied the impact of free-form communication on trust and trustworthiness. Charnness and Dufwenberg (2010) conducted the games with identical designs, except players were only given two restricted messages to choose from, one stated "I promise to choose Roll" ("bare promises"), while the other was blank. The impact on trust and trustworthiness was weaker with restricted as compared to free-form communication. Cooper and Kühn (2014) explore how 
communication fosters cooperation ${ }^{4}$. They find free-form chat improves cooperation much more than limited communication. Also, Cooper and Kühn (2015) investigates whether making it easier for subjects to reach an agreement by allowing more rounds of restricted communication can increase cooperation. Their results indicate that restricted communication is not a good substitute for the use of chat even after allowing subjects to have more rounds of interaction.

In broad brush-stroke, one message of the empirical literature is that the efficiency of economic outcomes is surprisingly high in the presence of pre-play communication, and more so when communication includes natural language. The question left unanswered is: why? Our paper, which points to the importance of richly-structured multi-meaning free-form messages, is another step towards answering this question.

\section{MODEL}

\subsection{Setup}

Two individuals are playing a two-stage game $\mathrm{G}^{*}(\mathrm{C}, \mathcal{G})$ where they can communicate in the first stage $\mathrm{C}$ and play a coordination game in the second stage $\mathcal{G}$. We call $\mathrm{G}^{*}(\mathrm{C}, \mathcal{G})$ an extended game of $\mathrm{G}$ if and only if players play $\mathrm{G}$ in stage $\mathcal{G}$, and correspondingly the game $\mathrm{G}$ is called the underlying game of $\mathrm{G}^{*}(\mathrm{C}, \mathcal{G})$.

In the below we focus on the case where the underlying game $\mathrm{G}$ is a symmetric coordination game with complete-information. Let the finite set $\mathrm{A}$ include all pure strategies (actions) $a_{i}$ of game $\mathrm{G}$. Let the set $\mathrm{S}$ includes all the mixed strategy $s_{i}$, where $s_{i} \in \Delta(A)$ is a randomization over the set $A$ of pure strategies. $U_{i}\left(a_{i}, a_{j}\right)$ is player $i$ 's payoff as a function of pure strategies and $\pi_{i}\left(s_{i}, s_{j}\right)$ is the expected payoff derived from $U_{i}(\cdot), i, j \in\{1,2\}, j \neq i$. The set $\boldsymbol{E}$ contains the Nash equilibria of game $G$. Nash equilibria are denoted as $E_{1}, \ldots, E_{K}$ and the strategies that constitute equilibrium $E_{k} \in \boldsymbol{E}$ are denoted as $\left(\mathrm{s}_{\mathrm{i}}^{\mathrm{E}_{\mathrm{k}}}, \mathrm{s}_{\mathrm{j}} \mathrm{E}_{\mathrm{k}}\right)$. Let $\boldsymbol{\varepsilon}$ be a subset of $\boldsymbol{E}$ containing Paretoefficient Nash equilibria, denoted by $\varepsilon_{1}, \ldots, \varepsilon_{K}$. The strategies that constitute a Pareto-efficient equilibrium $\varepsilon_{k} \in \mathcal{E}$ are denoted by $\left(s_{i}^{\varepsilon_{k}}, s_{j}^{\varepsilon_{k}}\right)$.

In the first stage $\mathrm{C}$, each player simultaneously sends a cheap-talk message $m_{i} \in M$, where $M$ is a publicly known nonempty message set. By the definition of cheap-talk, messages in $\mathrm{M}$ are

\footnotetext{
${ }^{4}$ Also called collusion in Cooper and Kühn (2014) context.
} 
non-binding, non-verifiable and costless. A function $\varphi: M \times M \rightarrow S$ specifies the second stage strategy $s_{i}=\varphi_{i}\left(m_{i}, m_{j}\right)$ given player $i$ sends message $m_{i}$ and receives $m_{j}, i \in\{1,2\}, j \neq i$.

Combining both stages, the finite set $A^{*}$ contains the pure strategies of $\mathrm{G}^{*}(\mathrm{C}, \mathcal{G}), a_{i}^{*}=$ $\left(m_{i}, \varphi_{i}\left(m_{i}, m_{j}\right)\right)$. A mixed strategy $\sigma_{i} \in \Delta\left(A^{*}\right)$ defines the probability assigned to pure strategy $A^{*} . \mathrm{V}_{i}\left(a_{i}^{*}, a_{j}^{*}\right)$ is player $i$ 's payoff as a function of pure strategies and $\Pi_{i}\left(\sigma_{i}, \sigma_{j}\right)$ is the expected payoff derived from $V_{i}(\cdot)$. We have $\Pi_{i}\left(\sigma_{i}, \sigma_{j}\right)=\pi_{i}\left(\varphi_{i}\left(m_{i}, m_{j}\right), \varphi_{j}\left(m_{j}, m_{i}\right)\right)$ given talk is costless. Furthermore, because communication is non-binding, any strategy $\left(\sigma_{i}, \sigma_{j}\right)$ which achieves a Nash equilibrium of $\mathrm{G}$ in the second stage $\mathcal{G}$ is a Nash equilibrium of $\mathrm{G}^{*}(\mathrm{C}, \mathcal{G})$, which we denote as $\left(\sigma_{i}^{E_{k}}, \sigma_{j}^{E_{k}}\right)$. Similarly, any $\left(\sigma_{i}, \sigma_{j}\right)$ which achieves a Pareto-efficient Nash equilibrium of $\mathrm{G}$ in the second stage $\mathcal{G}$ is a Pareto-efficient Nash equilibrium of $\mathrm{G}^{*}(\mathrm{C}, \mathcal{G})$, $\left(\sigma_{i}^{\varepsilon_{k}}, \sigma_{j}^{\varepsilon_{k}}\right)$

\subsection{Language Space}

We assume players share a common language space $M$, which consists of (1) a meaningful vocabulary; (2) a shared understanding among players that it is appropriate to interpret statements according to their literal meaning ${ }^{5}$. We focus on the focal meaning of messages, a concept first suggested by Farrell $(1993)^{6}$. We extend Farrell and Rabin's analysis ${ }^{7}$ to formally discuss the meaning of messages in two dimensions, and add another restriction, Attitude Ranking. We start by defining the E-meaning (equilibrium) and A-meaning (attitude) of messages as follows:

DEFINITION 1: The E-meaning of a message refers to the equilibrium that the sender signals her intention to play. We denote the set of messages that signal Nash equilibrium $E_{k}$ as $Q\left(E_{k}\right)$, $\forall k \in\{1, \ldots, K\}$.

\footnotetext{
${ }^{5}$ It is important to assume players communicate with common language not only because it eliminates the inessential multiplicity of equilibria in cheap-talk games, but also because it restricts the plausible interpretations of out-of-equilibrium messages.

${ }^{6}$ In Farrell (1993), the meaning of messages are expanded to a rich pre-existing common language set, where as long as the message's literal meaning is clear and only Credibility restricts communication.

${ }^{7}$ Farrell and Rabin's analysis refers to Farrell (1993) and Rabin (1994).
} 
DEFINITION 2: The A-meaning of a message indicates the strength of a player's desire to have her message followed by others. We denote the set of messages conveying attitude $\mathcal{A}_{w}$ as $D\left(\mathcal{A}_{w}\right), \forall w \in\{1, \ldots, W\}$.

We denote $\mathcal{A}$ as the set containing all possible A-meanings players can signal in the communication stage C. Suppose $\mathcal{A}$ includes $\mathrm{W}$ elements, which are denoted as $\mathcal{A}_{1}, \ldots, \mathcal{A}_{W}$ respectively. It is easy to see that, in the case where the set $\mathcal{A}$ is empty or it only contains only one element, it is equivalent for players either to play a two-dimension (A-meaning and $E$ meaning) or one-dimension (E-meaning only) cheap-talk game. Therefore, we concentrate on the cases where $W \geq 2$.

We continue by defining a complete language space both in E-meaning and A-meaning ${ }^{8}$. First, denote by $M\left(\mathrm{G}^{*}\right)$ the language space players use to communicate during the communication stage of game $\mathrm{G}^{*}(\mathrm{C}, \mathcal{G})$.

DEFINITION 3: The common language space $M\left(G^{*}\right)$ is complete with respect to its E-meaning if and only if:

(1) For all $m_{i} \in M$, there exists an equilibrium $E_{k} \in \boldsymbol{E}$ of $G$ such that $m_{i} \in Q\left(E_{k}\right)$;

(2) For any two equilibria $E_{k} \in \boldsymbol{E}, E_{l} \in \boldsymbol{E}$ and $k \neq l, Q\left(E_{k}\right) \cap Q\left(E_{l}\right)=\emptyset$.

DEFINITION 4: The common language space $M\left(G^{*}\right)$ is complete with respect to its A-meaning if and only if:

(1) For all $m_{i} \in M$, there exists attitude $\mathcal{A}_{w} \in \mathcal{A}$ such that $m_{i} \in D\left(\mathcal{A}_{w}\right)$;

(2) For all attitudes $\mathcal{A}_{w} \in \mathcal{A}, \mathcal{A}_{y} \in \mathcal{A}$ and $w \neq y, D\left(\mathcal{A}_{w}\right) \cap D\left(\mathcal{A}_{y}\right)=\emptyset$.

Part (1) of Definition 3 says that all messages contain an E-meaning, and Part (2) illustrates that no message can simultaneously signal two different equilibria. Analogously, Definition 4 says that all messages convey an A-meaning, and that no single message can convey more than one attitude.

\footnotetext{
${ }^{8}$ Rabin (1994) defines a complete and common space for the E-meaning of language. We extend this idea to another dimension of language - attitude.
} 
In the development below, following Rabin $(1994)^{9}$, we assume players' messages consist solely of proposals to play Pareto-efficient Nash equilibria.

\subsection{Equilibria}

\subsubsection{Agreement-equilibrium}

We now move to how players form an "agreement" in a two-dimensional language space. Rabin (1994) says players have reached an agreement when they mention the same equilibrium in the pre-play communication stage. We extend the notion of agreement into a language space with both E-meaning and A-meaning, and proceed to define the concept of an agreementequilibrium.

ASSUMPTION 1 (Credibility): If it is optimal for a player to honor the E-meaning of her own message given the other player honors the E-meaning of her own message, then the E-meanings of messages from both players will be honored.

Roughly put, Credibility assumes players won't deviate from their words if there is no incentive for them to so. ${ }^{10}$

PROPOSITION 1: Paired players jointly play $\varepsilon_{k}$ in the second stage if they made proposal $\boldsymbol{m}=\left(m_{1}, m_{2}\right)$, where $m_{i} \in Q\left(\mathcal{E}_{k}\right), i \in\{1,2\}, \forall \mathcal{E}_{k} \in \mathcal{E}$ of underlying game $G$.

If paired players signal a shared intention to play the same Pareto-efficient Nash equilibrium of underlying game $\mathrm{G}$ then, by the assumption of Credibility, they have an implicit agreement to play the signaled equilibrium. This type of equilibrium of $\mathrm{G}^{*}$ is called an agreement-equilibrium and can be characterized as follows:

DEFINITION 5: $A$ set $\left(\sigma_{1}, \sigma_{2}\right)$ where $\sigma_{i} \equiv\left(m_{i}, s_{i}\right), \forall i \in\{1,2\}$, is called agreement-equilibrium of game $G^{*}(C, \mathcal{G})$ iff

\footnotetext{
${ }^{9}$ This is referred to as "Pareto talk" by Rabin (1994). Rabin considers this one of the simplest examples of negotiating language, where it consists solely of proposals to play Pareto-efficient Nash equilibria.

${ }^{10}$ Farrell $(1988,1993)$ summarizes the intuition of Credibility as "communication cannot work well when there are incentives to lie". Demichelis and Weibull (2008) add a lexicographic preference for honesty (small costs of lying) to their model to capture the similar idea.
} 
(1) Players make proposal set $\boldsymbol{m}=\left(m_{1}, m_{2}\right)$ in stage $C$, where $m_{i} \in Q\left(\varepsilon_{k}\right) \forall i \in\{1,2\}$;

(2) Players play $\varepsilon_{k}=\left(s_{1}^{\varepsilon_{k}}, s_{2}^{\varepsilon_{k}}\right)$ in stage $\mathcal{G}$.

\subsubsection{Negotiated-equilibrium}

We continue by considering how a message's $A$-meaning can be used to achieve equilibrium when paired players signal the intention to play different equilibria.

ASSUMPTION 2 (Attitude Ranking): Suppose players share a complete and transitive ordering of the elements in $\mathcal{A}$. Given $m_{i} \in Q\left(\varepsilon_{k}\right) \cap D\left(\mathcal{A}_{w}\right)$ and $m_{j} \in Q\left(\varepsilon_{l}\right) \cap D\left(\mathcal{A}_{y}\right), i, j \in$ $\{1,2\}, j \neq i$, if $\mathcal{A}_{w}$ indicates stronger desire of having the message followed than $\mathcal{A}_{y}$, then players will play $\varepsilon_{k}$ in stage $\mathcal{G}$.

We assume that players share a complete and transitive ordering for A-meanings. This requires players have the same interpretation of attitude with the preexisting common language. For example, we assume that everyone in the game agrees that the message "Let's do X no matter what" expresses a stronger desire to have the message followed than a message like "It's up to you, if you have no strong opinion, let's do X".

Assumption 2 says that if there are two different $E$-meanings and two different $A$-meanings in the two messages from paired players, then both players will honor the E-meaning of the message which includes the A-meaning indicating stronger desire to have one's message followed. We are now in position to explain how simultaneous "negotiated agreement" can occur within a single round of simultaneous multi-way communication. Given Attitude Ranking, players are able to use A-meanings to resolve the conflicts between E-meanings.

DEFINITION 6: $A$ set $\left(\sigma_{1}, \sigma_{2}\right)$, where $\sigma_{i} \equiv\left(m_{i}, s_{i}\right), \forall i \in\{1,2\}$, is called negotiatedequilibrium of game $G^{*}(C, \mathcal{G})$ iff

(1) Player i makes a proposal which satisfies $m_{i} \in Q\left(\varepsilon_{k}\right) \cap D\left(\mathcal{A}_{w}\right)$ in stage $C$, and player $j$ makes a proposal $m_{j}$ such that $m_{j} \notin Q\left(\varepsilon_{k}\right)$ but $m_{j} \in D\left(\mathcal{A}_{y}\right)$, where $\mathcal{A}_{w}$ indicates stronger desire to the message followed than $\mathcal{A}_{y}$;

(2) Players play $\varepsilon_{k}=\left(s_{1}^{\varepsilon_{k}}, s_{2}^{\varepsilon_{k}}\right)$ in the stage $G$. 


\subsubsection{Communication-failure}

We close the analysis of equilibria by describing the case where different E-meanings but the same A-meaning are contained in the messages. That is, neither the conditions of agreementequilibrium nor negotiated-equilibrium are achieved with pre-play communication.

ASSUMPTION 3 When $m_{i} \in Q\left(\varepsilon_{k}\right) \cap D\left(\mathcal{A}_{w}\right)$ and $m_{j} \in Q\left(\varepsilon_{l}\right) \cap D\left(\mathcal{A}_{w}\right)$, the message set $\boldsymbol{m}=\left(m_{i}, m_{j}\right)$ will be ignored, $i, j \in\{1,2\}, i \neq j, \forall \varepsilon_{k} \in \mathcal{E}, \varepsilon_{l} \in \mathcal{E}$ and $\varepsilon_{k} \neq \varepsilon_{l} \forall \mathcal{A}_{w} \in \mathcal{A}$.

Assumption 3 says that when paired players' messages convey different $E$-meanings but the same A-meaning, since it is not possible to coordinate based on the message.

DEFINITION 7: A set $\left(\sigma_{1}, \sigma_{2}\right)$, where $\sigma_{i} \equiv\left(m_{i}, s_{i}\right)$, is called communication-failure for game $G^{*}(C, \mathcal{G})$ if

(1) For $m=\left(m_{1}, m_{2}\right), m_{i} \in Q\left(\varepsilon_{k}\right) \cap D\left(\mathcal{A}_{w}\right), m_{j} \in Q\left(\varepsilon_{l}\right) \cap D\left(\mathcal{A}_{w}\right), \forall \varepsilon_{k} \in \mathcal{E}, \varepsilon_{l} \in$ $\mathcal{E}$ and $\varepsilon_{k} \neq \varepsilon_{l} \forall \mathcal{A}_{w} \in \mathcal{A}$.

(2) Players play the game as if there is no communication stage $C$.

Communication-failure does not necessarily lead to coordination failure. Definitions 5, 6 and 7 illustrate all possible message-combinations that paired players are able to send in cheaptalk environments where messages have two dimensions of meaning.

\subsection{Communication Strategies}

We now move to the analysis of communication strategies in the first stage. The assumptions we made allow us to restrict attention to only three different communication situations, i.e. signaling same E-meaning, signaling different E-meanings with different $A$ meanings, and signaling different E-meanings with same A-meaning. Suppose it is common knowledge that everyone responds to messages based on these assumptions. Given players' beliefs about what messages they might receive, there exist ex-ante best messaging strategies. However, the strategies highly depend on the payoff structure of the underlying coordination game. For ease of exposition, we concentrate on pure coordination games since that the identical payoffs for two players isolate the function of cheap talk as a pure coordination device as 
compared to its use for any other information revealing purposes (e.g., indicating different marginal utility of money, or different social preferences or risk attitudes). Note it is straightforward to generalize this framework to other environments.

In a pure coordination game where players are indifferent among all Pareto-efficient Nash equilibria, it restricts attention to communication strategies meant to avoid communication failure. We denote the two pure-strategy Pareto-efficient equilibria as $\varepsilon_{k}$ and $\varepsilon_{l}$, and one Paretodominated mixed-strategy equilibrium as $\varepsilon_{m}$. To simply the characterization of equilibria characterization, we assume there are exactly two attitudes available, $\mathcal{A}_{w}$ and $\mathcal{A}_{y}$.

PROPOSITION 2: When the underlying game $G$ is a pure coordination game, in which equal payoffs of pure-strategy Nash equilibria, if a player believes that she is least likely to receive message $m_{j} \in Q\left(\varepsilon_{l}\right) \cap D\left(\mathcal{A}_{w}\right), i \in\{1,2\}, j \neq i, k \neq l$, then she maximizes her expected payoff by sending message $m_{i} \in Q\left(\varepsilon_{k}\right) \cap D\left(\mathcal{A}_{w}\right)$.

Proposition 2 says that in order to maximize expected utility in a cheap-talk game $\mathrm{G}^{*}$ with a pure coordination game with the equal payoff for two pure-strategy Nash equilibrium, one needs to minimize the chance of encountering $\varepsilon_{m}$ by sending a message with a different $E$-meaning but the same A-meaning of the message one is least likely to receive.

The only time players cannot achieve a Pareto-efficient outcome is when they face communication-failure. For each combination of A-meaning and E-meaning player $j$ can send, there is a corresponding combination of A-meaning and E-meaning that leads to communicationfailure. Player $i$ desires to minimize the chance of communication-failure given her belief over the distribution her counterparts' messages. ${ }^{11}$

\section{AN EXAMPLE: MODIFIED HOLM (2000) PINK-BLUE GAME}

Our interest is in determining whether people use and respond to natural language messages in a way that is consistent with the model above. To do this, following Holm (2000) ${ }^{12}$, we design

\footnotetext{
${ }^{11}$ Further, notice that the more categories of attitude there are, the less possible paired players encounter failure. If attitude is continuous in one language, even a language with only two-dimensional meanings, i.e. E-meaning and $A$-meaning, could achieve full coordination situation.

${ }^{12}$ Holm (2000) discusses the idea that introducing Pink and Blue labels into Battle of Sexes Game where gender is revealed information might improve coordination rate.
} 
a "Pink-Blue" Game, where options are labeled as Pink and Blue, and counterparts' genders are revealed to players at beginning. We use an announcement to ensure it is common knowledge that, for the purpose of this game, "Pink is a color often preferred by females, and Blue is a color often preferred by males". To the Holm (2000) environment, we added a pre-play free-form communication stage $\mathrm{C}$, where players are able to send each other simultaneous messages. After the message exchange, players make decisions in the Pink-Blue game, the payoff matrix for which is described by Figure 1. As indicated by the Figure 1, players earn $x>0$ if they choose the same color, and zero otherwise. We adopt the game to create two different situations, both when there exists a focal point (when players of the same gender are matched) and when there does not exist a focal point (when players of different genders are matched) before communication. We want to investigate how communication plays a role in each of those two cases.

Figure 1. Modified Holm (2000) Pink-Blue Game

\begin{tabular}{|c|c|c|c|}
\hline Player 1 & & & \\
\hline & & Blue & Pink \\
\hline & Blue & $x, x$ & 0,0 \\
\hline & Pink & 0,0 & $x, x$ \\
\hline
\end{tabular}

The Pink-Blue game has three Nash equilibria: two pure-strategy equilibria (Pink, Pink), (Blue, Blue) as well as a mixed-strategy equilibrium where each player plays each action with probability 0.5 . Neither of the pure-strategy equilibrium Pareto-dominates the other, but both dominate the mixed strategy equilibrium. Given that Pink-Blue is a complete information game, players can use pre-play communication exclusively to make claims about intended moves in order to break the symmetry (as compared to, for example, using this stage to reveal private information ${ }^{13}$ ).

Next, we map the Pink-Blue game to the framework described above. We begin by specifying the language space with two possible Pareto-efficient Nash equilibria $\mathcal{E}=\{$ (Blue, Blue)

\footnotetext{
${ }^{13}$ Farrell $(1987,1988)$ and Rabin $(1991,1994)$ provide formal analysis of communication as a means to convey intentions and thereby improve coordination among rational players. Farrell $(1987,1988)$ studies signaling intentions in simple sequential games of complete information, where an underlying game is preceded by one or more rounds of structured pre-play communication. Rabin $(1991,1994)$ extends analysis of Farrell $(1987,1988)$ and studies coordination in a more general class of games with multiple rounds of communication.
} 
(Pink, Pink) $\}$ as E-meanings, and two possible $A$-meanings $\mathcal{A}=\{$ Demanding, Deferring $\}$, where demanding indicates stronger desire of have the message followed than deferring.

HYPOTHESIS 1: Players communicate with one of four types of messages in game $G^{*}(C, \mathcal{G})$ : Demanding Blue (DmB), Demanding Pink (DmP), Deferring Blue (DfB), Deferring Pink (DfP). i.e. $m_{i} \in M=\{D m B, D m P, D f B, D f P\}, \forall i \in\{1,2\}$.

$D m B$ refers to the case where the sender intended to play Blue, and requests the other player also play Blue; $D f B$ refers to the case where the sender intended play Blue, but is ultimately deferring the choice to the player, and analogously for $D m P$ and $D f P$. Hypothesis 1 is built on Definition 1-4. It implies that players in Pink-Blue Game communicate in this well-defined common and complete space with both E-meaning and A-meaning.

\subsection{Equilibria of Pink-Blue}

Based on Assumption 1-3 and Definition 5-7, we obtain following three hypotheses and summarize all connections between messages and actions in Figure 2. When two players signal the same color, they can achieve an agreement-equilibrium. When two players signal different colors with different attitudes, they achieve a negotiated-equilibrium. Communication failure occurs when the two players signal different colors with same Attitude. In this case, they ignore the messages and play a mixed-strategy Nash equilibrium.

HYPOTHESIS 2: If paired players send messages signaling intentions to the same color, they will play that color.

HYPOTHESIS 3: If paired players send messages signaling intentions to play different colors with one Demanding and one Deferring attitudes, both the demanding player and the deferring players will play the color the demanding player signaled. 
HYPOTHESIS 4: If paired players send messages signaling intentions to play different colors but with the same attitude, they will play mixed strategies with half chance choosing Blue, and half choosing Pink.

Figure 2. Responses with Two Conditions in Modified Pink-Blue Game

\begin{tabular}{|c|c|c|c|c|}
\hline $\mathrm{Player} i$ & $D m B$ & $D f B$ & $D m P$ & DfP \\
\hline$D m B$ & (Blue, Blue) & (Blue, Blue) & $\left(\left(\frac{1}{2}, \frac{1}{2}\right),\left(\frac{1}{2}, \frac{1}{2}\right)\right)$ & (Blue, Blue) \\
\hline$D f B$ & (Blue, Blue) & (Blue, Blue) & (Pink, Pink) & $\left(\left(\frac{1}{2}, \frac{1}{2}\right),\left(\frac{1}{2}, \frac{1}{2}\right)\right)$ \\
\hline$D m P$ & $\left(\left(\frac{1}{2}, \frac{1}{2}\right),\left(\frac{1}{2}, \frac{1}{2}\right)\right)$ & (Pink, Pink) & (Pink, Pink) & (Pink, Pink) \\
\hline$D f P$ & (Blue, Blue) & $\left(\left(\frac{1}{2}, \frac{1}{2}\right),\left(\frac{1}{2}, \frac{1}{2}\right)\right)$ & (Pink, Pink) & (Pink, Pink) \\
\hline
\end{tabular}

\subsection{Communication Strategies}

Figure 2 illustrates the links between all 16 possible paired communication situations and response actions. In Pink-Blue game, a pair of players is guaranteed to coordinate on a Nash equilibrium in the second stage as long as both players respond to each communication situation in first stage according to our model's three assumptions. However, one presumably wants to avoid Pareto-dominated outcomes that result from communication failure. This can be done by optimally use E-meaning and A-meaning of messages based on beliefs over the distribution of message types.

HYPOTHSIS 5: With a focal point of (Blue, Blue) in the second stage game, players will send messages with Blue E-meaning, and with a focal point of (Pink, Pink) in the second stage game, players will send messages with Pink E-meaning.

HYPOTHESIS 6: Absent a focal point in the second stage game (male-female group), players will send messages including different E-meaning and same A-meaning of messages they believe least likely to receive. 
Hypothesis 6 is derived directly from Proposition 3. Hypothesis 5 is based on the fact that focal point is a solution that people will tend to use in the absence of communication, because it seems natural, special, or relevant to them ${ }^{14}$. When adding a communication stage to games with natural focal points, and thus high rates of coordination in the absence of communication, the improvements communication can make may be limited. Players are nevertheless able to use communication as way to confirm expectations. If both players are females, (Pink, Pink) is focal, while if both are males (Blue, Blue) is focal. With same-gender groups, the focal point is clear given we made announcement about the color-gender relevance, the role of communication in this case is only to confirm. While in a mixed-gender groups, communication can create a focal meaning, once paired players agree on which equilibrium to play, there is no incentive to deviate.

\section{EXPERIMENTAL TEST}

We conduct Modified Holm (2000) Pink-Blue game with no communication (baseline treatment), with restricted communication allowing one-dimensional and two-dimensional signaling and with free-form communication.

In baseline, the game proceeds as follows. We begin by randomly assigning people to pairs. We then reveal the gender of each player to the counterpart. Finally, the players simultaneously choose a color. If they choose the same color, then both earn the same amount of money in addition to their show-up payment. Otherwise, both earn only the show-up amount.

Figure 3. Instruction Table

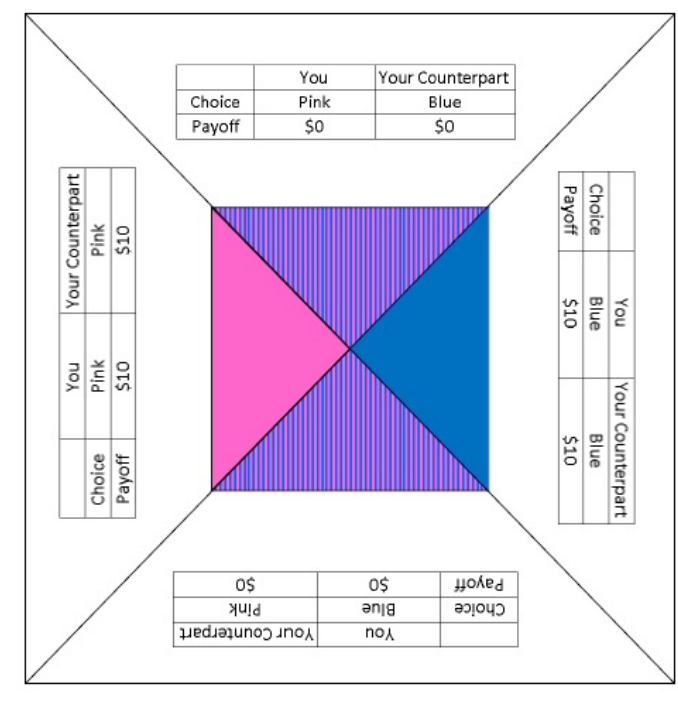

\footnotetext{
${ }^{14}$ See more at Schelling (1960), p. 57
} 
To avoid menu effects (e.g. the possibility that participants will coordinate on the first listed option), we use a presentation without default "first option". In particular, we use a square payoff figure (Figure 3) that ensures no option is more salient than any other.

To ensure color-labels were treated the same way across sessions and cultures, in all instructions subjects were informed: "Pink is a color often preferred by females, and Blue is a color often preferred by males".

All the treatments with communication proceed just as in baseline, with the exception that every subject has one chance to send a message to her counterpart simultaneously. They are not able to read the counterparts' notes until they have finished writing their own messages. Messages are written and delivered after the gender of the counterpart has been revealed and prior to subjects' Pink/Blue choices. The details of the messages subjects can send in each communication treatment are summarized below.

\subsection{Treatments}

In the restricted communication treatment with one-dimensional meaning, subjects are informed they can choose one message from two to communicate with their counterparts: "I am choosing Pink" or "I am choosing Blue".

In the restricted communication treatment with two-dimensional meaning, subjects are informed that each of them can choose one message from four to communicate with their counterparts: "I am choosing Pink no matter what", "I am choosing Blue no matter what", "I am choosing the color your message tells me to. If your message says the same, I am choosing Pink." or "I am choosing the color your message tells me to. If your message says the same, I am choosing Blue."

In the free-form communication treatment, each subject is given a piece of blank paper. They are told they can write anything except identification information about themselves to their counterpart to communicate.

\subsection{Procedures}

We use a between-subject design. That is, each subject experiences only exactly one treatment. In all treatments, each subject plays three rounds of the same game and faces different counterparts each round. At the beginning of each round, subjects are randomly and 
anonymously paired. The randomization is not constrained by gender, though most of our subjects experienced games with both genders. At the end of the experiment, one of the three rounds was randomly selected to determine subjects' payoffs.

During the experiment players are seated at separated computer terminals and are given a copy of the experiment's instructions. These instructions are also read aloud, as we want to ensure subjects understand that the information contained in them is common knowledge.

All four treatments have been conducted at George Mason University, Washington, D.C. The baseline and free-form communication treatments were also conducted in Shanghai Jiao Tong University, Shanghai. Subjects interact only with people from the same university. At Mason, subjects were paid $\$ 5$ for showing up and $\$ 10$ for coordination success. At SJTU, subjects were paid $10 \mathrm{RMB}$ for showing up and $40 \mathrm{RMB}$ for coordination success ( 1 US dollar $\approx$ $6.14 \mathrm{RMB}$ and $1 \mathrm{US}$ dollar $\approx 6.06 \mathrm{RMB}$ when we conducted the experiment in Shanghai, May 2013 and Dec. 2013) The experiment lasted about 40 minutes and the average payoff was designed to be slightly above the local hourly wage for subjects from the two subject pools.

\section{RESUTLS}

Subjects were recruited from undergraduate populations at George Mason University (GMU) and Shanghai Jiao Tong University (SJTU). Each of the 342 participations made 3 decisions, for a total of 1026 observations. Table 1 summarizes our subjects' demographics.

Table 1 - Demographics of Subjects in Two Pools

\begin{tabular}{lcc}
\hline \hline & George Mason Univ. & Shanghai Jiao Tong Univ. \\
& Washington D.C., U.S. & Shanghai, China \\
\hline Number of Subjects & 208 & 134 \\
Percentage of Males & $49.04 \%$ & $64.18 \%$ \\
Average Age & 20.63 & 21.83 \\
Average GPA & 3.24 & 3.35 \\
\hline
\end{tabular}

There are few things need to be mentioned about our subjects pool. First, given our focus is natural language communication, we ran baseline and free-form communication treatments both in GMU and SJTU, while two restricted treatments were only conducted in GMU. Second, the gender ratio of SJTU is unbalanced, which reflects the university unbalanced gender ratio. This is probably due to that SJTU is a University famous for its engineering majors. Third, about Seventy percent of participants at GMU were born in United States, and all of the SJTU 
participants were born in China. Finally, the age and GPA of GMU and SJTU are comparable to each other.

\subsection{Baseline and Restricted Communication}

RESULT 1. Restricted communication with both one-dimensional and two-dimensional meaning facilitates coordination. Restricted communication with two-dimensional meaning facilitates coordination more than one-dimensional meaning.

Figure 4 illustrates the coordination rate in three treatments: baseline (i.e. no communication), restricted communication with one-dimensional meaning (abbreviated as 1D Restricted Communication in the figures) and restricted communication with two-dimensional meaning (abbreviated as 2D Restricted Communication in the figures). The coordination success is significantly improved by both restricted communication with one-dimensional meaning (Wilcoxon Mann-Whitney (henceforth, WMW) test: $\mathrm{z}=-2.898, \mathrm{P}=0.004$, two-sided test, $n_{1}=$ $126, n_{2}=174$ ) and restricted communication with two-dimensional meaning (WMW test: $\mathrm{z}=$ 4.845, $\mathrm{P}<0.001$, two-sided test, $n_{1}=126, n_{2}=168$ ). Moreover, the improvement on coordination rate with restricted communication with two-dimensional meaning is significant higher than restricted communication with one-dimensional meaning (WMW test: $\mathrm{z}=-2.182$, $\mathrm{P}=0.029$, two-sided test, $n_{1}=174, n_{2}=168$ ).

Figure 4. Coordination Rate - Baseline, 1D Restricted and 2D Restricted

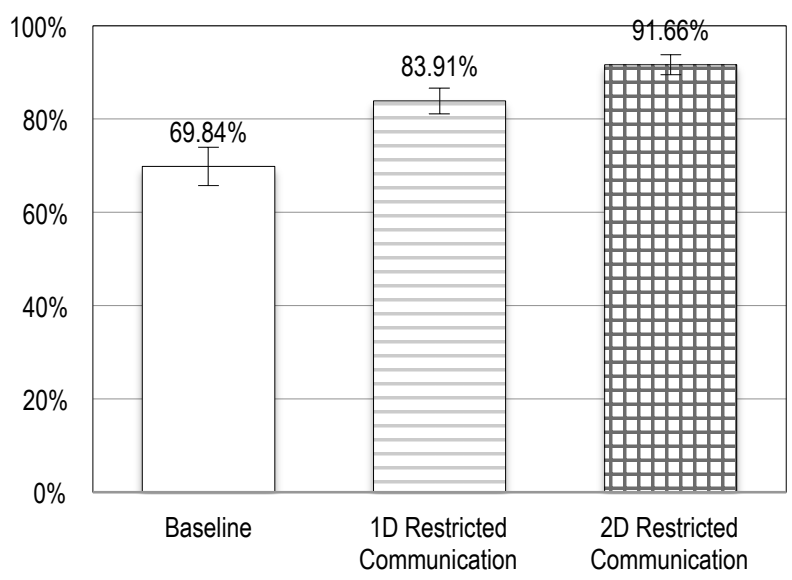

RESULT 2. More improvement of coordination is made with mixed-gender groups than singlegender groups. 
Figure 5 breaks down the coordination rate into single-gender pairs and mixed-gender pairs. While twelve percent of coordination rate improvement is made by communication with onedimensional meaning in mixed-gender groups, only six percent of coordination improvement with same-gender groups. Twenty-four percent of coordination improvement is made by communication with two-dimensional meaning in mixed-gender groups, while only eleven percent of coordination improvement with same-gender groups. With mixed-gender groups, restricted communication with two-dimensional meaning has significantly higher coordination success than restricted communication with one-dimensional meaning (WMW test: $\mathrm{z}=-1.680$, $\mathrm{P}=0.093$, two-sided test, $n_{1}=76, n_{2}=70$ ); no significant difference has been found in singlegender groups (WMW test: $\mathrm{z}=-1.440, \mathrm{P}=0.150$, two-sided test, $n_{1}=98, n_{2}=98$ ). The intuition behind these results, of course, is that coordination rates are already high with same-gender groups without any communication given the existence of focal points.

Figure 5. Coordination Rate with and without Restricted Communication
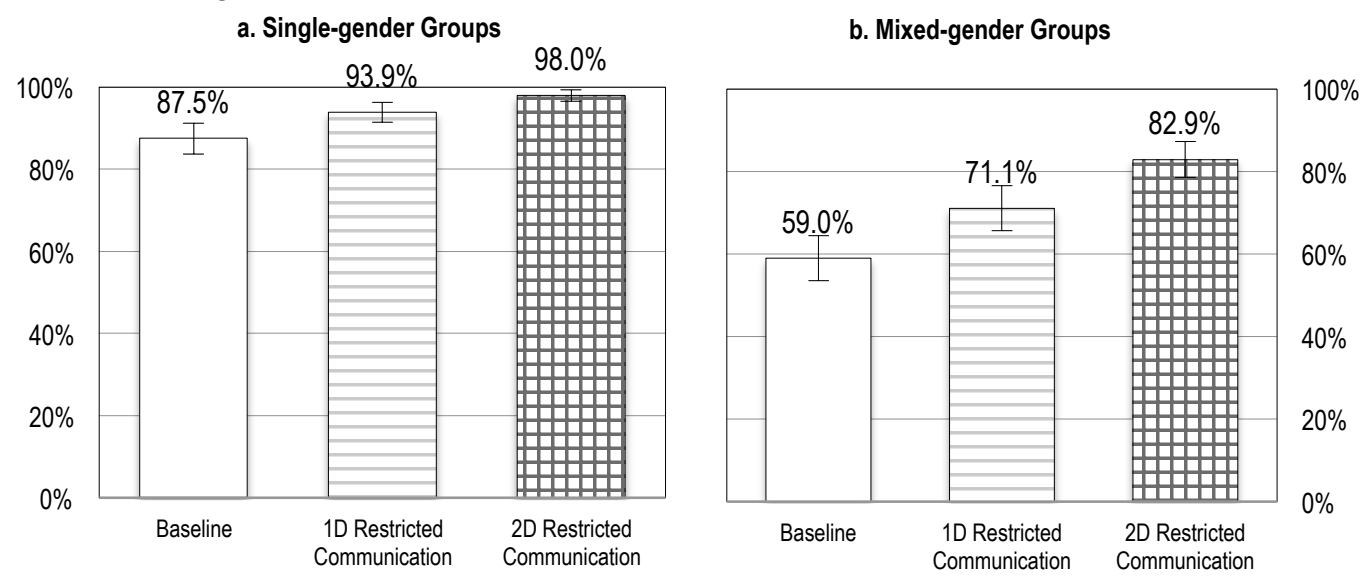

\subsection{Free-form Communication}

We conducted baseline and free-form communication treatments in both Shanghai Jiaotong University and George Mason University ${ }^{15}$. In this subsection, the data from both subjects pools will be discussed.

\footnotetext{
${ }^{15}$ One important reason we tested the model in both China and U.S. is to investigate the robustness of results about free-form communication across language.
} 
RESULT 3. Free-form communication significantly improves coordination rates from baseline in both subject pools. The improvement is significant in mixed-gender groups, not significant in same-gender groups.

Let's first compare the coordination rates between the baseline and free-form communication treatment in joint subjects pool. The coordination rate is significantly increased under communication treatment (WMW test: $\mathrm{z}=-4.271, \mathrm{P}<0.001$, two-sided test, $n_{1}=$ $100, n_{2}=128$ ). This result holds if we test the same hypothesis respectively in the two subject pools (WMW test: $\mathrm{z}=-4.173, \mathrm{P}<0.001$, two-sided test, $n_{1}=42, n_{2}=52$ in GMU; $\mathrm{z}=-1.707$, $\mathrm{P}=0.088$, two-sided test, $n_{1}=58, n_{2}=76$ in SJTU). Further, while the coordination rate is increased significantly by free-form communication in mixed-gender groups (Wilcoxon MannWhitney test: $\mathrm{z}=-4.796, \mathrm{P}<0.001$, two-sided test, $n_{1}=56, n_{2}=66$ ), the change in same-gender groups is insignificant (WMW test: $\mathrm{z}=1.197, \mathrm{P}=0.23$, two-sided test, $n_{1}=44, n_{2}=62$ ). The intuition, again, is that coordination rates are already very high in same-gender groups in the baseline treatment.

\subsubsection{Nature of Free-form Communication}

In order to understand further what subjects write in the free-form messages, we analyze the content of messages. We use the Houser and Xiao $(2011)^{16}$ procedure to classify our messages. We recruit 32 evaluators from SJTU and 37 from GMU, who have never participated in our experiment, to categorize the free-form messages we collected. Each message is both in its initial form and translated into Chinese (or English), and classified by these third party evaluators from both pools. Evaluators read messages and place them into five categories: "Demanding Pink", "Deferring Pink", "Demanding Blue", "Deferring Blue" and "None of Above". In the instructions, they were told: 'you should choose 'Demanding Pink' if you believe the message writer intends to play Pink, and requests that her or his counterpart also chooses Pink. You should choose 'Deferring Pink' if you believe the message writer intends to Pink, but is ultimately deferring the choice to her or his counterpart." Similarly for "Demanding Blue" and

\footnotetext{
${ }^{16}$ While some previous literature on communication uses research assistants as evaluators, Houser and Xiao (2011) discuss the advantage of using a coordination game classify message content. This classification method is increasingly used to classify messages from free-form communication studies (e.g. Xiao and Houser, 2005; Gächter et al. 2013; Deck et al. 2013 ; Ong et al. 2012; Chen et al. 2014).
} 
"Deferring Blue". Evaluators are paid based on whether their categorizations of three random chosen messages are consistent with most popular categorizations for that session.

To provide a sense for the nature of messages in each category, in the following we provide one sample message from each category (see all 384 messages and their classifications at online Appendix).

One message was, "Pick Blue no matter what". $97.30 \%$ of American evaluators and $87.50 \%$ of Chinese evaluators classified this message as "Demanding Blue".

Other subjects wrote: "Hi, I will follow what you wrote on this paper. If you signaled Pink, I will choose Pink, if you wrote Blue, I will choose Blue. If you didn't signal any color, please choose Blue." 94.59\% American evaluators and 96.87\% Chinese evaluators classify this message as "Deferring Blue".

"Pink! Girl's Rule! No if ands or but! I will NOT change! 100\% Pink!! I will not choose otherwise even if you $100 \%$ say different or tell me to change! I will NOT change! Go Pink!” $89.19 \%$ of American evaluators and $93.75 \%$ of Chinese evaluators classify this message as "Demanding Pink".

"Going with whatever color you say. (If you don't mention a color I will go with Pink.)" $91.89 \%$ of American evaluators and $96.88 \%$ of Chinese evaluators classify this message as "Deferring Pink".

A final example is: "The point of coming to these experiments is to make money. I don't care about the colors and what-not. So let's try and choose the same option." $97.30 \%$ of American evaluators and $96.88 \%$ of Chinese evaluators classify this message as "None of Above".

We use the classification of a message as one of the four categories in our model only if more than $50 \%$ of the evaluators from both pools place it in that same category. We classify a message as "None of Above" if either more than half of evaluators from both pools believe it belongs to "None of above", or fewer than $50 \%$ of evaluators agree on any of the four named categories.

RESULT 4. 95.83\% of cases messages are classified consistently by evaluators from the U.S. and China as communicating with one of the four types: Demanding Blue, Deferring Blue, Demanding Pink or Deferring Pink. 
Figure 6 shows the distribution of classifications among all the messages we collected from the two subjects pools. Among the 384 free-form messages collected, only ten messages were categorized differently between Shanghai subjects and D.C. subjects, and only six messages were not classified within one of the four named classifications. Thus, $95.83 \%$ of messages were classified consistently by evaluators from both pools as one of the four named communication types. Result 4 is a test of Hypothesis 1.

Figure 6. Number of Messages Classified as Each Communication Style (By Culture)

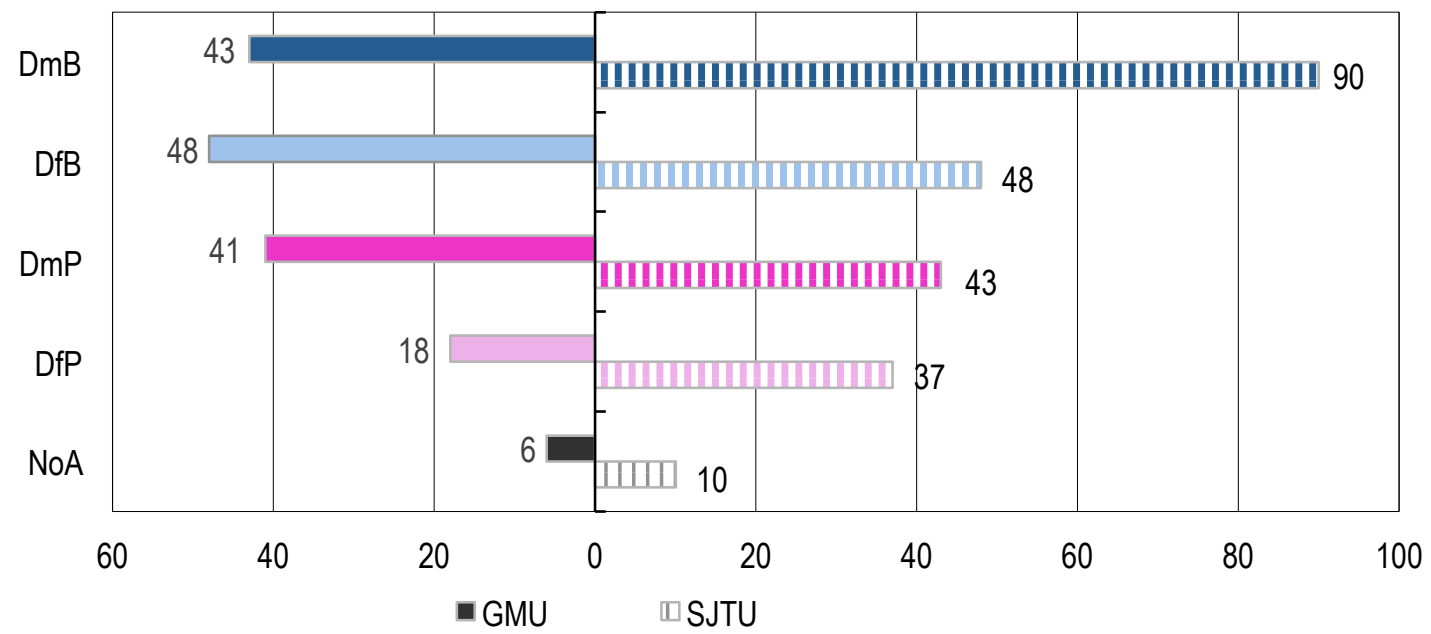

It is important to emphasize that messages were classified based on message content only. Evaluators were provided no other information. In particular, evaluators did not know which messages were paired and knew neither the gender nor the choices of the message writers. Because evaluations are independent of participants' choices, we are able to use the classified $E$ meanings and A-meanings to test whether people wrote and responded to messages as our theory predicts.

\subsubsection{Responses to Free-form Messages}

In this subsection, we focus on analysis of cases where both messages from a pair of players are classified as one of the four named types (which is $95.83 \%$ of the messages we collected). We test all the hypotheses about how people respond to paired messages. 
RESULT 5. When paired players send messages that signal same color, in $99.6 \%$ of cases they choose to honor the color in messages. There is neither significant cultural nor gender differences in how people respond to messages. Overall, the coordination rate is as high as $99.2 \%$.

We confirm the validity of Hypothesis 2 through Result 5. Overall, in $99.6 \%$ of cases players choose to honor their messages when both players signaled the same pure-strategy equilibrium. As a result, players achieve agreement-equilibrium in $99.2 \%$ of the cases. As shown in Figure $7 \mathrm{a}$ and Figure 7b, there is no significant difference in how many people chose to honor their words both cross-culture and between genders ${ }^{17}$.

Figure 7. Fraction of Players Chose to Honor the E-meaning (Color) of Messages

a. Fraction of Players Chose to Honor the E-meaning (Color) of Messages (By Culture)

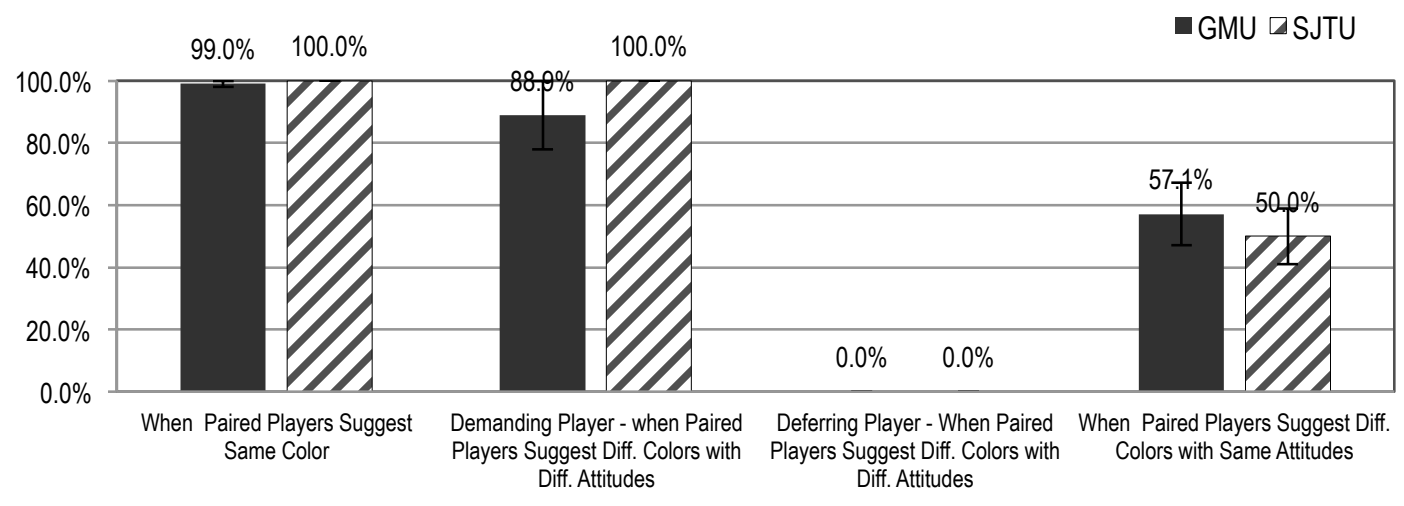

b. Fraction of Players Chose to Honor the E-meaning (Color) of Messages (By Gender)

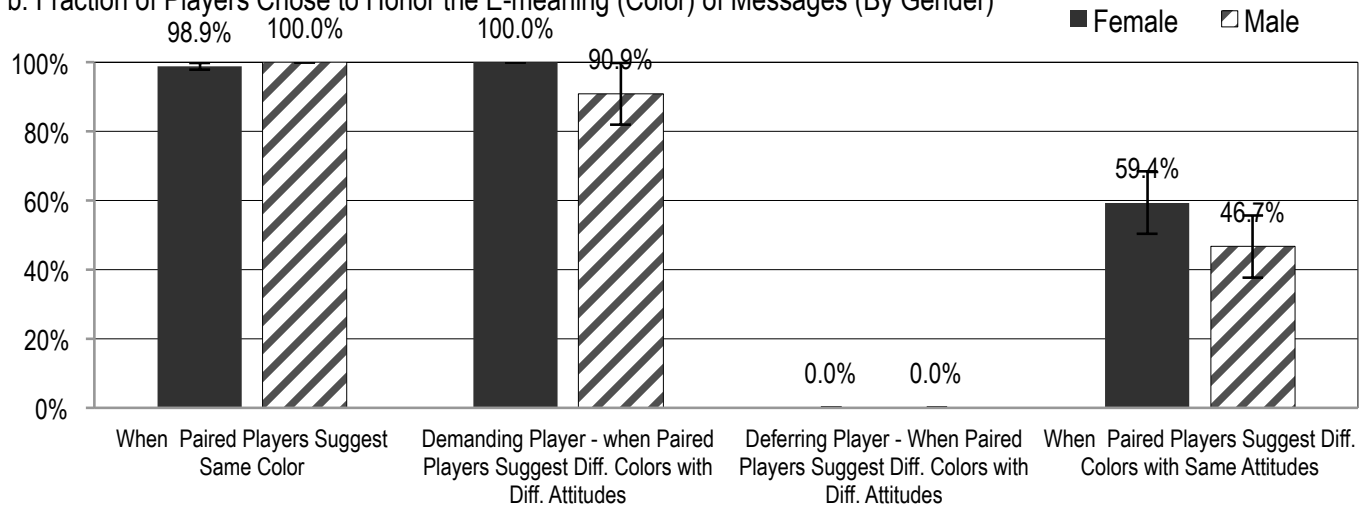

\footnotetext{
${ }^{17}$ Regarding the independence of the data included in our non-parametric tests, only the first observation from each individual in each comparison category is included. For both cross culture and between gender comparisons, the rate of honoring one's stated color is $100 \%$ for each pool respectively. Thus, no $\mathrm{P}$-value is provided here.
} 
RESULT 6. When paired players send messages that signal different colors, and one of them sends a demanding message, and the other sends a deferring message, in $95.7 \%$ of cases demanding players choose to honor their the color in the messages, while no deferring player chooses to honor the color in their message. Further, GMU players respond to attitude as well as SJTU players, and male players respond to attitude as well as female players.

Empirically, pooled across locations, in $95.7 \%$ of 232 cases demanding players choose to honor their words and $100 \%$ of deferring players switch to demanding players' announced option. As a result players reach negotiated-equilibrium in $95.7 \%$ of the cases where it is possible. This is strong evidence people do take advantage of and respond to the A-meaning of language. As depicted in Figure 7a, the cultural difference of neither how demanding players respond (WMW test: $\mathrm{z}=-1.173, \mathrm{P}=0.241$, two-sided test, $n_{1}=8, n_{2}=11$ ) nor how deferring players respond in this case is not significant (WMW test ${ }^{18}: \mathrm{z}=$., $\mathrm{P}=$., two-sided test, $n_{1}=6, n_{2}=12$ ). As shown in Figure $7 \mathrm{~b}$, the gender difference of neither how demanding players respond (WMW test: $\mathrm{z}=0.949, \mathrm{P}=0.343$, two-sided test, $n_{1}=9, n_{2}=10$ ) nor how deferring players respond in this case if not significant (WMW test: $\mathrm{z}=$., $\mathrm{P}=$., two-sided test, $n_{1}=12, n_{2}=6$ ).

RESULT 7. When paired players send messages that signal different colors with same attitude, in $53.2 \%$ of cases players choose to honor the color in the messages, and this fraction is not significantly different across cultures or genders.

As is shown in Figure 7, the fraction of people who choose to honor their words when paired players signaled different colors with same attitude is around $50 \%$, and this number is consistent across cultures (WMW test: $\mathrm{z}=0.441, \mathrm{P}=0.659$, two-sided test, $n_{1}=22, n_{2}=27$ ) and gender (WMW test: $\mathrm{z}=0.704, \mathrm{P}=0.481$, two-sided test, $n_{1}=25, n_{2}=24$ ).

When paired players send messages including different E-meanings and the same $A$ meaning, about half of the subjects choose to honor the color in their messages. However, the overall coordination rate is still as high as $87.1 \%$, which implies that they find other ways to coordinate. Our suspicion is that the attitudes subjects indicate in the messages have more than

\footnotetext{
${ }^{18}$ For both cross-culture and between gender comparisons of deferring players' responses, all players chose to honor demanding players' E-meanings. Thus, no P-value can be provided.
} 
just two categories (i.e. demanding or deferring), which we were not able to test with our current classification.

RESULT 8. When the conditions of agreement equilibrium or negotiated equilibrium are achieved in the first stage, coordination success in the second stage is significantly higher than otherwise.

Given that conditions of which equilibrium players have reached after the first stage is a result of their own communication style as well as their counterpart's communication style rather than a randomly assigned independent variable, we should not perform non-parametric tests. To accommodate the non-independence issue with respect to individuals, we ran OLS econometric models which control for random effects at individual level. The dependent variable in the model is coordination success, which takes value of 1 if two matched players choose the same option, while takes a value of zero otherwise. The independent variables included in the regressions are: negotiated equilibrium condition dummy, which takes value of 1 if the subjects have achieved the conditions for negotiated equilibrium in the first stage, and 0 otherwise; communicationfailure condition dummy, which takes value of 1 if subjects face the condition of communication failure in the first stage, and 0 otherwise; Culture dummy, which takes a value of one for GMU subjects, and 0 for SJTU subjects; Gender dummy, which takes a value of 1 for male subjects, and 0 for female subjects; Gender of counterpart dummy, which is equal to 1 for the subjects who interact with male counterparts in the round, and 0 for the subjects who interact with female counterparts; age, year of staying the current location, GPA, and relationship status, which takes a value of 1 if subjects are committed in a relationship, takes a value of 2 if subjects has a relationship but not committed to it, takes a value of 3 if single.

Model 1 of Table 3 includes data from both males and females and both locations. The first regression shows that the coordination rate under baseline of agreement equilibrium condition, after controlled for culture, gender, gender of counterpart, age, year at one's current location, GPA, relationship status, is significantly higher than communication failure cases, while not significantly different than negotiated equilibrium cases.

Model 2-4 pairwise compare when conditions of different equilibria are achieved. Model 2 only includes data from cases when the conditions of either agreement equilibrium or negotiated 
equilibrium have been achieved. Model 3 only includes data of cases when the conditions of either negotiated equilibrium or communication-failure equilibrium have been achieved. Model 4 only includes data from cases when the conditions of either agreement equilibrium or communication-failure equilibrium have been achieved.

These regressions show that (i) the coordination rate in cases when agreement equilibrium conditions have been achieved is higher than when negotiated equilibrium conditions have been achieved; (ii) the coordination rate in cases when either agreement equilibrium conditions or negotiated conditions have been achieved is significantly higher than when communicationfailure conditions have been achieved.

Table 3 - Effect of Achievement of Equilibrium Conditions on Coordination Rate

\begin{tabular}{|c|c|c|c|c|}
\hline \multicolumn{5}{|c|}{ Dependent Variable: Coordination Success } \\
\hline Model & 1 & 2 & 3 & 4 \\
\hline \multirow[t]{2}{*}{ Negotiated Equilibrium Conditions } & -0.02 & $-0.04^{* *}$ & & \\
\hline & $(0.03)$ & $(0.02)$ & & \\
\hline \multirow[t]{2}{*}{ Communication-failure Conditions } & $-0.11^{* * *}$ & & $-0.12^{* *}$ & $-0.11^{* * *}$ \\
\hline & $(0.03)$ & & $(0.06)$ & $(0.03)$ \\
\hline \multirow[t]{2}{*}{ Culture } & 0.03 & $-0.06^{*}$ & -0.04 & -0.00 \\
\hline & $(0.03)$ & $(0.03)$ & $(0.07)$ & $(0.03)$ \\
\hline \multirow[t]{2}{*}{ Gender } & 0.02 & -0.00 & $0.16^{\star *}$ & 0.03 \\
\hline & $(0.02)$ & $(0.02)$ & $(0.07)$ & $(0.02)$ \\
\hline \multirow[t]{2}{*}{ Gender of Counterpart } & 0.02 & -0.01 & $0.16^{\star *}$ & 0.03 \\
\hline & $(0.02)$ & $(0.01)$ & $(0.07)$ & $(0.02)$ \\
\hline \multirow[t]{2}{*}{ Age } & 0.00 & 0.00 & 0.00 & 0.01 \\
\hline & $(0.01)$ & $(0.01)$ & $(0.01)$ & $(0.01)$ \\
\hline \multirow[t]{2}{*}{ Year } & -0.00 & 0.00 & -0.00 & -0.00 \\
\hline & $(0.00)$ & $(0.00)$ & $(0.00)$ & $(0.00)$ \\
\hline \multirow[t]{2}{*}{ GPA } & -0.03 & -0.02 & $-0.15^{\star}$ & -0.02 \\
\hline & $(0.02)$ & $(0.03)$ & $(0.08)$ & $(0.02)$ \\
\hline \multirow[t]{2}{*}{ Relationship Status } & 0.01 & 0.03 & 0.01 & 0.01 \\
\hline & $(0.01)$ & $(0.01)$ & $(0.04)$ & $(0.01)$ \\
\hline \multirow[t]{2}{*}{ Constant } & $0.92^{* * *}$ & $0.88^{* * *}$ & $1.20^{* * *}$ & $0.90^{* * *}$ \\
\hline & $(0.16)$ & $(0.17)$ & $(0.43)$ & $(0.17)$ \\
\hline Number of Observations & 343 & 283 & 104 & 299 \\
\hline
\end{tabular}




\subsubsection{Communication Strategies with Free-form Communication}

Next, we investigate the communication strategies players use in different gender pairings and across cultures. Figure 8 partitions the distribution of communication styles into the four possible pairings (Male-Male, Female-Female, Male-Female, Female-Male) and two cultures (GMU, SJTU).

RESULT 9. Male-Male matched players send messages with $D m B$ and $D f B$ communication styles more frequently than DmP and DfP. Female-Female matched players send message with $D m P$ and $D f P$ communication style more frequently than $D m B$ and $D f B$.

\section{Figure 8. Communication by Matching Type and University}

8a. Male Sent to Male Messages

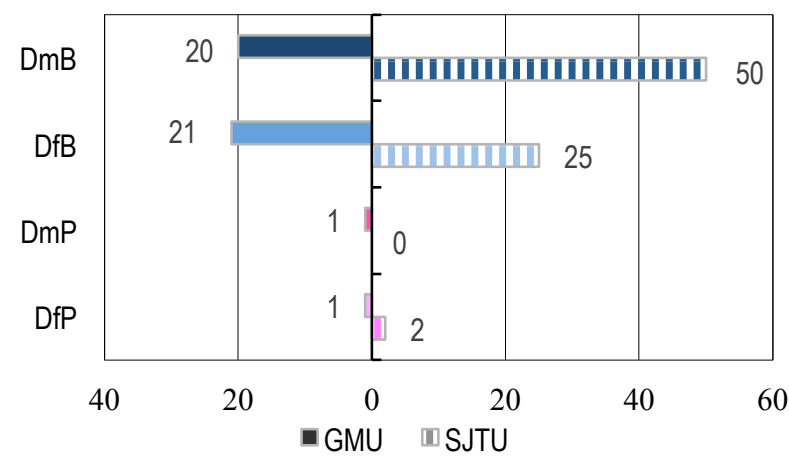

8c. Male Sent to Female Messages

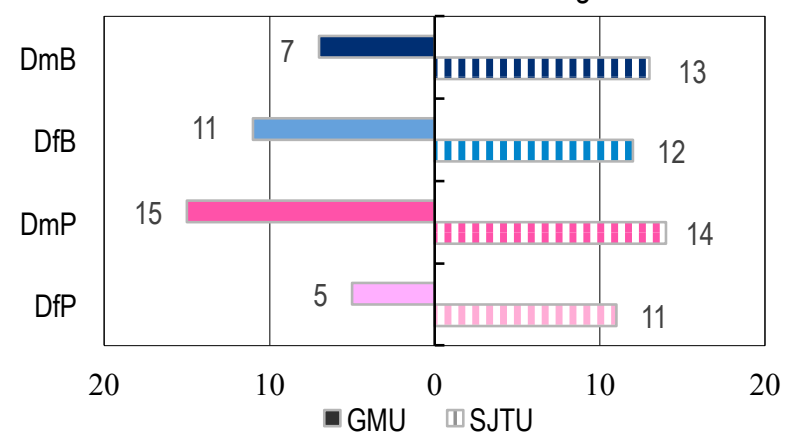

8b. Female Sent to Female Messages

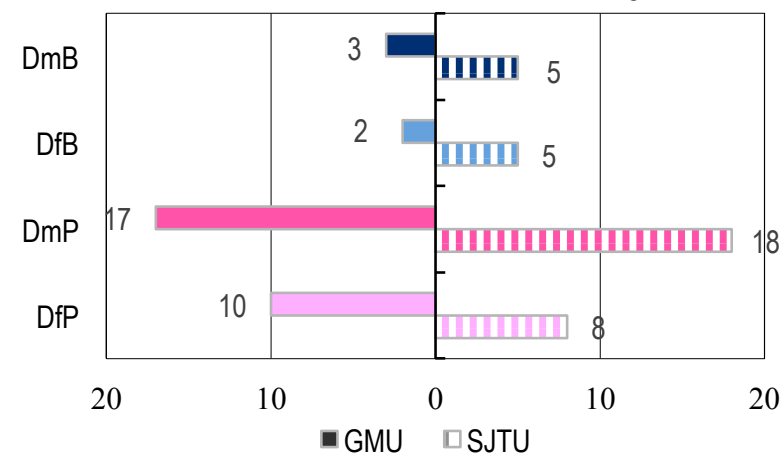

8d. Female Sent to Male Messages

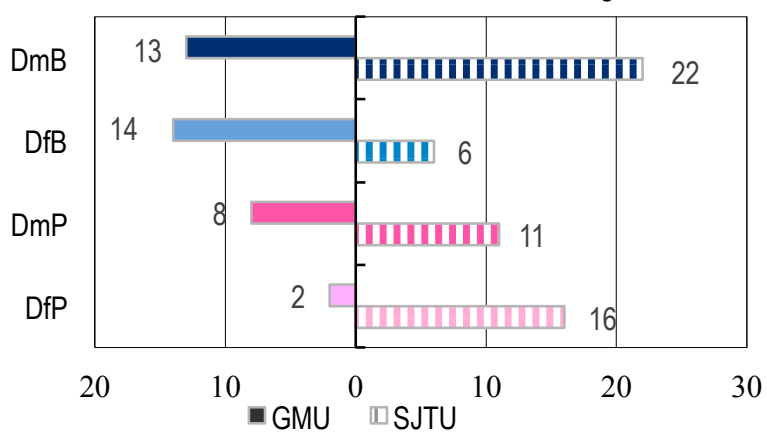

As Figure 8a shows, only $4.7 \%$ of cases in GMU and $2.6 \%$ of cases in SJTU Male-Male matched players choose communication style with Pink E-meaning. Slightly more Blue Emeaning messages are sent by females, but still much less than Pink E-meaning messages. $15.6 \%$ of cases in GMU and $27.7 \%$ of cases in SJTU Female-Female players choose communication styles with Blue E-meaning (see Figure 8b). Comparing part $\mathrm{c}$ and part $\mathrm{d}$ of 
Figure 8, one can observe some gender differences in communication styles in the mixed-gender environment, which we will address in next result.

In order to investigate more about the gender differences of communication styles, we adopt a statistical classification procedure proposed by El-gamal and Grether (1995). With this procedure, we are able to group all the messages collected from each individual to investigate her communication styles. Based on the observation that some subjects always send the same style of message regardless the gender of the counterpart, while the others vary the E-meaning or $A$ meaning according to their counterpart's gender, we assume in our statistical model that players in the game include exclusively behavioral types chosen randomly from separate distributions, each assigning positive prior probability to four "dogmatic" types, as well as to a sophisticated type ${ }^{19}$.

Specifically, we restrict attention to following types of players: $\operatorname{dogmatic} D m B$ players, who always send $D m B$ message; dogmatic $D f B$ players, who always send $D f B$ message; dogmatic $D m P$ players, who always send $D m P$ message; dogmatic $D f P$ players, who always sends $D f P$ message; and sophisticated players, who send the messages with focal point color as E-meaning if there is a focal point (same-gender pairings), while send messages with different E-meanings and same A-meaning of least popular communication style among the counterparts' population if there is no focal point (mixed-gender pairings) ${ }^{20}$.

For each subject $i$, we calculate a sequence of communication style statistics $x_{1}^{i}, \ldots, x_{T_{i}}^{i}$, for each trial based on the classification of her messages and counterpart's gender. $x_{c s^{h}, \tau}^{i} \in$ $\left\{x_{d D m B, \tau}^{i}, x_{d D f B, \tau}^{i}, x_{d D m P, \tau}^{i}, x_{d D f P, \tau}^{i}, x_{s o p h, \tau}^{i}\right\}$, where $x_{c S^{h}, \tau}^{i}$ equals 1 if the subject i's decision on trial $\tau$ agrees with rule $h$, and zero otherwise. We observe $T_{i}$ decisions for a subject $i$. Now we define the sufficient statistic, $X_{c s^{h}}^{i}=\sum_{\tau=1}^{T_{i}} x_{c s^{h}, \tau}^{i}$ (the number of decisions that agree with rule $h$ ). Following El-Gamal and Grether (1995), we assume different subjects may use different rules and the error rate is same for all subjects and all tasks. We introduce the possibility that subjects make errors with probability $\varepsilon$. This allows each of our decision rules to give a positive probability (likelihood) to all possible patterns of behavior. The likelihood function for subject $i$ is:

\footnotetext{
${ }^{19}$ We are inspired by Crawford (2003), which assumes players' roles in a sender-receiver game are filled by players who chosen randomly from four possible mortal types (boundedly rational types), as well as to a sophisticated type.

${ }^{20}$ Given our experiment design, we assume that (Blue, Blue) is focal if two male subjects are matched, while (Pink, Pink) is focal if two female subjects are matched, and there is no focal point for mixed-gender matches.
} 


$$
f^{c s, i}\left(x_{1}^{i}, \ldots, x_{T_{i}}^{i}\right)=\left(1-\frac{\varepsilon}{2}\right)^{X_{c s}^{i}} \times\left(\frac{\varepsilon}{2}\right)^{\left(T_{i}-X_{c s}^{i}\right)}
$$

If there are $I$ subjects, each indexed by $i$, then a natural way to estimate the communication style is to apply maximum likelihood using:

$$
(\widehat{c S}, \hat{\varepsilon})=\operatorname{argmax}_{c s, \varepsilon} \prod_{i=1}^{I} \prod_{h=1}^{H}\left(f^{c s^{h}, i}\left(x_{1}^{i}, \ldots, x_{T_{i}}^{i}\right)\right)^{\delta_{i h}}
$$

where $\delta_{i h} \in\{0,1\}$ equals 1 if subject $i$ is using rule $h$, and zero otherwise. In summary, we

Figure 9. Communication Styles Classification by El-gamal and Grether (1995) Method (By Gender)

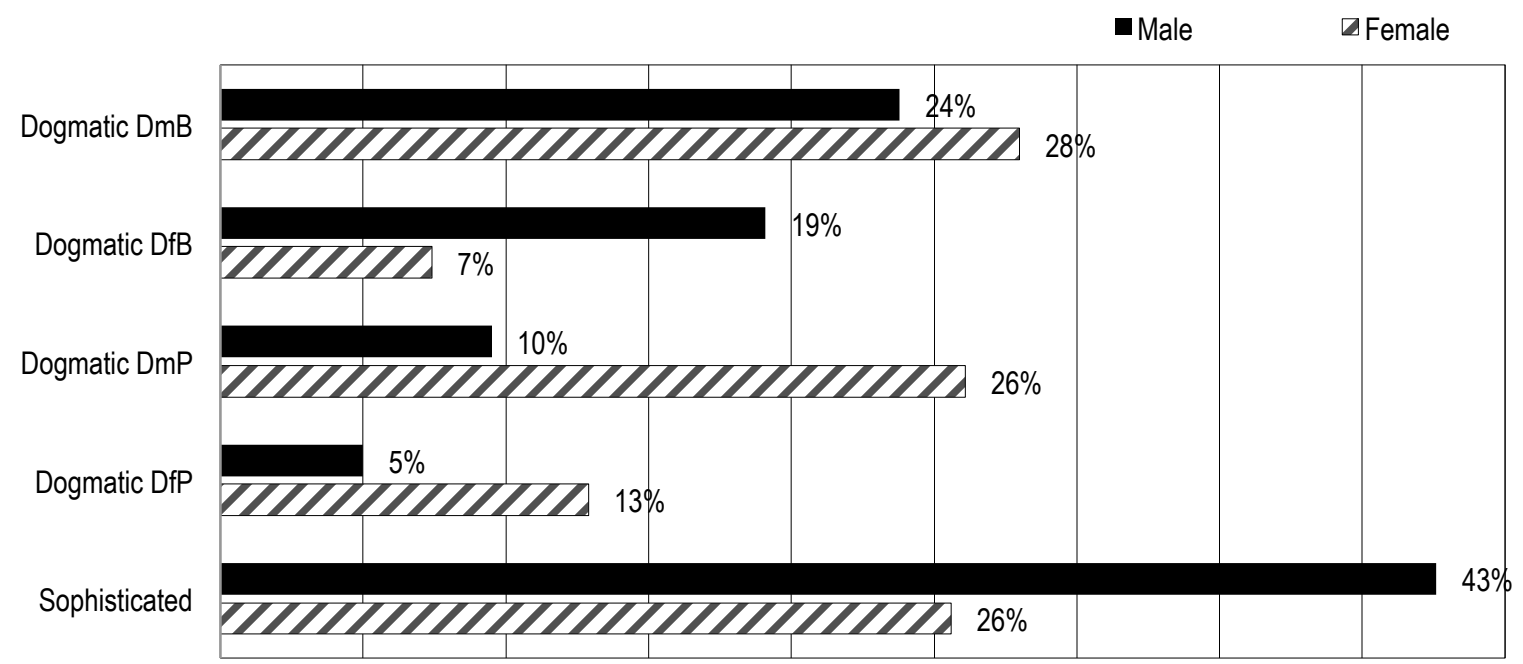

first calculate the maximum likelihood $f^{c s^{h}, i}$ of each communication rule $c s^{h}$ for each individual $i$; we choose the communication rule to maximize $f^{c s, i}$ for each individual $i$; we multiply the obtained likelihood over individual $i \in\{1, \ldots, I\}$, and maximize the outcome by choosing $(\widehat{c S}, \hat{\varepsilon})$.

Turning on to estimation results, Figure 9 summarizes the outcome of El-gamel and Grether (1995) procedure when applied to our data. Each bin bar represents to percentage of 128 subjects fit into the communication styles.

RESULT 10. Besides a large proportion of players are using sophisticated communication styles, males are more likely be dogmatic senders who always send Blue messages, while females are more likely to be dogmatic senders who always send demanding messages. 
As shown in Figure 9, 43\% of male players are sophisticated senders and $26 \%$ of females are sophisticated senders. Further, while $43 \%$ of male players are either dogmatic DmB senders or $D f B$ senders, only $15 \%$ of male players are dogmatic Pink message senders. $54 \%$ of females are dogmatic demanding senders while only $20 \%$ of females are dogmatic deferring senders.

RESULT 11. Coordination rates are not only affected by the E-meaning of the messages, but also by their A-meaning. When only male subjects are included, coordination rate is only affected by E-meaning. While when only female subjects are included, coordination rate is only affected by A-meaning.

Table 4 shows five OLS regressions, all of which control for random effects at the individual level. The dependent variable is coordination success, which takes value of 1 if two matched players choose the same option, and is zero otherwise. In addition to the controls included in Table 3, we also include an E-meaning dummy, taking value of 1 (0) if a player signals Blue (Pink), and an A-meaning dummy taking value 1 (0) if a player is demanding (deferring).

As Model 1 of Table 3 shows, when nearly all messages ${ }^{21}$ are included, both the E-meaning and A-meaning impact coordination rates significantly (controlled for culture, gender, gender of counterpart, age, year, GPA, and relationship status). Generally speaking, both sending messages with Pink E-meaning and communicating with a demanding attitude increase the chance of coordination success. For males (Model 2), only E-meaning has an effect, while for females (Model 3), only A-meaning has an effect. With mixed-gender pairs (Model 4 for males, Model 5 for females), only demanding messages from females seem to improve coordination.

\footnotetext{
${ }^{21} 28$ observations do not show in the regression because we are lack of some of the control dummies (mostly GPA).
} 
Table 4 - Effect of Communication Strategies on Coordination Rate

\begin{tabular}{|c|c|c|c|c|c|}
\hline \multicolumn{6}{|c|}{ Dependent Variable: Coordination Success } \\
\hline Model & 1 & 2 & 3 & 4 & 5 \\
\hline \multirow[t]{2}{*}{ E-meaning of messages } & $-0.05^{* *}$ & $-0.05^{*}$ & -0.06 & -0.04 & 0.02 \\
\hline & $(-0.03)$ & $(-0.03)$ & $(0.04)$ & $(0.05)$ & $(0.05)$ \\
\hline \multirow[t]{2}{*}{ A-meaning of messages } & $0.06^{* * *}$ & 0.01 & $0.14^{* * *}$ & -0.01 & $0.11^{* *}$ \\
\hline & $(0.02)$ & $(0.02)$ & $(0.04)$ & $(0.05)$ & $(0.05)$ \\
\hline \multirow[t]{2}{*}{ Culture } & 0.01 & -0.01 & 0.02 & 0.03 & $0.12^{*}$ \\
\hline & $(0.03)$ & $(0.04)$ & $(0.06)$ & $(0.08)$ & $(0.07)$ \\
\hline \multirow[t]{2}{*}{ Gender } & $0.06^{\star *}$ & & & & \\
\hline & $(0.02)$ & & & & \\
\hline \multirow[t]{2}{*}{ Gender of Counterpart } & $0.05^{\star *}$ & $0.06^{* *}$ & -0.05 & & \\
\hline & $(0.02)$ & $(0.02)$ & $(0.04)$ & & \\
\hline \multirow[t]{2}{*}{ Age } & 0.01 & 0.01 & -0.00 & $0.02^{*}$ & -0.02 \\
\hline & $(0.01)$ & $(0.01)$ & $(0.01)$ & $(0.01)$ & $(0.01)$ \\
\hline \multirow[t]{2}{*}{ Year } & -0.00 & -0.00 & -0.00 & -0.01 & 0.00 \\
\hline & $(0.00)$ & $(0.00)$ & $(0.00)$ & $(0.00)$ & $(0.00)$ \\
\hline \multirow[t]{2}{*}{ GPA } & -0.01 & -0.03 & 0.00 & -0.05 & -0.06 \\
\hline & $(0.03)$ & $(0.03)$ & $(0.05)$ & $(0.06)$ & $(0.06)$ \\
\hline \multirow[t]{2}{*}{ Relationship Status } & 0.02 & 0.02 & 0.02 & 0.02 & $0.08^{* *}$ \\
\hline & $(0.01)$ & $(0.01)$ & $(0.03)$ & $(0.03)$ & $(0.03)$ \\
\hline \multirow[t]{2}{*}{ Constant } & $0.80^{* * *}$ & $0.90^{* * *}$ & $0.76^{* *}$ & $0.77^{* *}$ & $1.00^{* * *}$ \\
\hline & $(0.18)$ & $(0.18)$ & $(0.37)$ & $(0.36)$ & $(0.42)$ \\
\hline Number of Observations & 356 & 205 & 151 & 86 & 88 \\
\hline
\end{tabular}

\subsection{Baseline, Restricted Communication and Free-form Communication}

Finally, we compare the different impact of all three communication treatments on coordination and look for the reasons why one works better than the other ${ }^{22}$.

RESULT 12. The coordination rate in free-form communication treatment is as high as Restricted Communication with two-dimensional meaning.

As shown in Figure 10, in the free-form communication treatment, the coordination rate is as high as $93.59 \%$, which is not significantly higher than restricted communication

\footnotetext{
${ }^{22}$ All the results in this subsection only have GMU data included since that SJTU subjects didn't participate the restricted communication treatments.
} 
with two-dimensional meaning (WMW test: $\mathrm{z}=-0.659 \mathrm{P}=0.510$, two-sided test, $n_{1}=$ $\left.168, n_{2}=156\right)$, but significantly higher than restricted communication with onedimensional meaning (WMW test: $\mathrm{z}=-2.747 \mathrm{P}=0.006$, two-sided test, $n_{1}=174, n_{2}=156$ ).

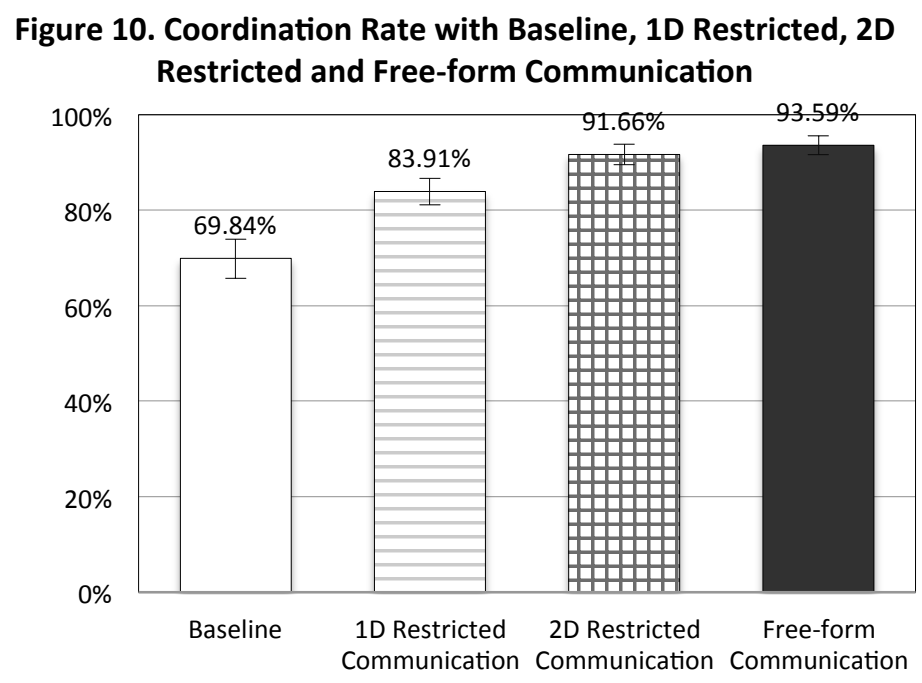

RESULT 13. When paired players send messages that signal the same color, the coordination rate is not different between free-form communication and restricted communication with twodimensional meaning. When paired players send messages that signal different colors with different attitudes, the coordination rate is slightly higher in restricted communication with twodimensional meaning. The free-form communication improves coordination significantly more than restricted communication with two-dimensional meaning when paired players send messages that signal different colors with the same attitude.

Figure 11 breaks down the coordination rate of restricted communication with twodimensional meanings and free-form communication into three different communication cases, when paired players signal same color, when paired players signal different colors with different attitudes, and when paired players signal different colors with same attitudes. In the first case, when E-meaning of the messages match, the coordination rate has no difference in two treatments (WMW test: $\mathrm{z}=1.500, \mathrm{P}=0.133$, two-sided test, $n_{1}=112, n_{2}=100$ ). When paired players signal different E-meanings with different A-meanings, restricted communication with two-dimensional meanings works slightly better than free-form communication (WMW test: $\mathrm{z}$ 
$=1.905, \mathrm{P}=0.057$, two-sided test, $\left.n_{1}=32, n_{2}=18\right)$. When paired players signal different $E$ meanings with same A-meaning, free-form communication works much better than restricted communication with two-dimensional meanings (WMW test: $\mathrm{z}=-3.296, \mathrm{P}=0.001$, two-sided test, $\left.n_{1}=24, n_{2}=28\right)$.

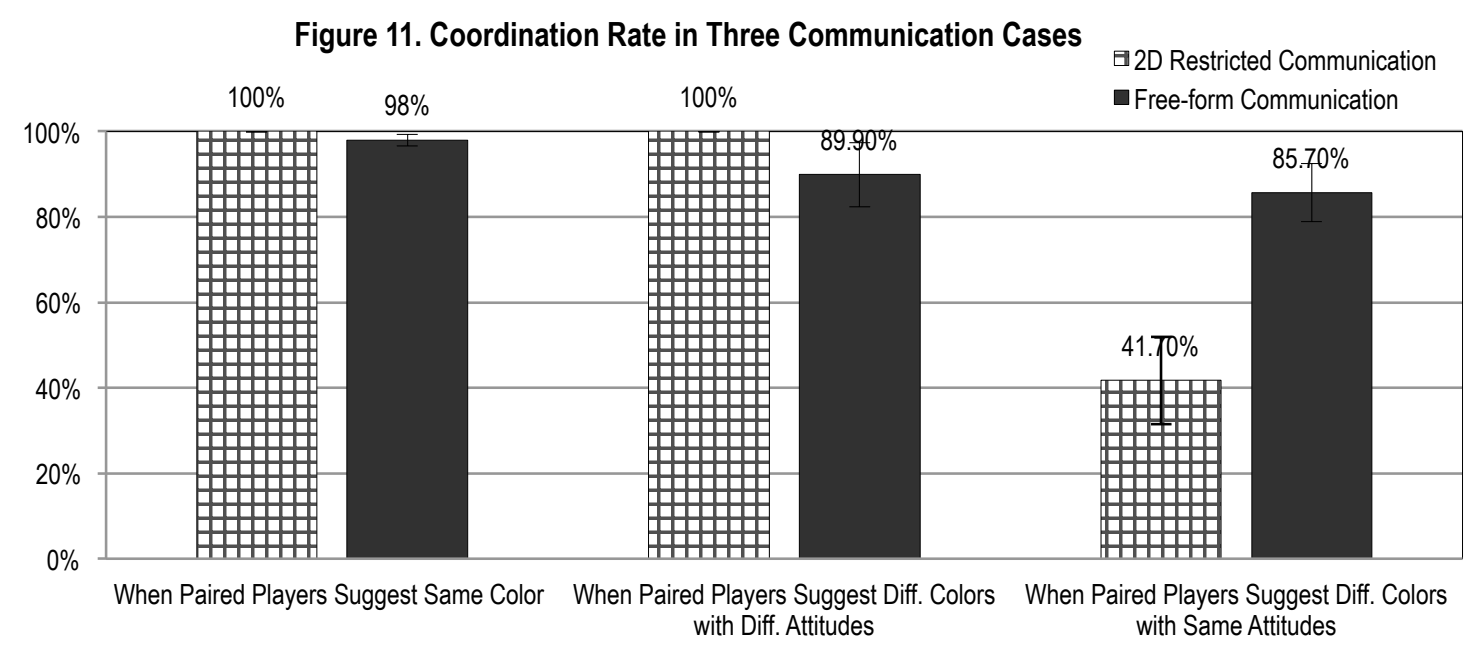

RESULT 14. When paired players send messages that signal different colors with same attitude, more of them honor the E-meaning (color) signaled in the messages in the free-form communication treatment than restricted communication with two-dimensional meanings.

We compare the fractions of players choose to honor the E-meaning of messages in 2dimension restricted communication with free-form communication treatment. Given no significant different patterns have been found in all other three cases in Figure 12 (i.e. when players signal same color, whether the demanding players honor the E-meaning when paired players signal different colors with different attitudes, whether the deferring players honor the $E$ meaning when paired players signal different colors with different attitudes), significantly less players honor the E-meaning of the messages in restricted communication with two-dimensional meanings treatment (WMW test: $\mathrm{z}=-2.005, \mathrm{P}=0.045$, two-sided test, $n_{1}=24, n_{2}=28$ ). 
Figure 12. Fraction of Players Chose to Honor the E-meaning (Color) of Messages

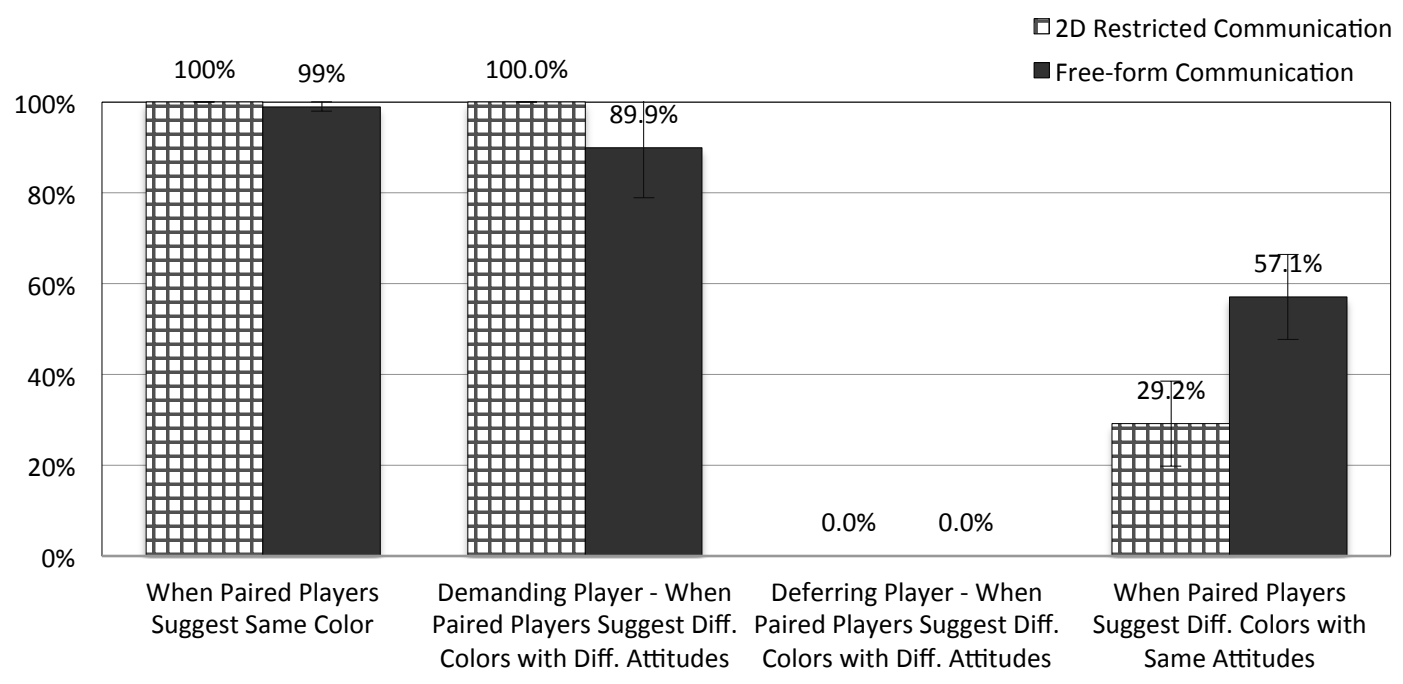

\section{CONCLUSIONS AND DISCUSSION}

This paper provides an explanation for why natural language communication promotes coordination better than classical intention signaling. Independent from intention signaling, we assume each player is able to signal the strength of desire to have her message followed. We show that, allowing two-dimensional signaling reduces the chance of coordination failure. Conducting a series of experiments, our findings confirm that when communicating in free-form, people write messages that include both signaled intentions and attitudes, and people respond to both when making their decisions. Consequently, free-form communication promotes coordination as well as restricted communication with two-dimensional meaning, and significantly better than one-dimensional intention signaling or games without communication. As Robert Gibbons mentioned in his book ${ }^{23}$ "The spirit of cheap talk is that anything could be said, but formalizing this would require $\mathrm{M}$ to be a very large set" Our results shed light on the fundamental question of how rich a message space should be in order closely to capture key features of cheap-talk.

Use of a predetermined restricted message space might have methodological advantages, for example by allowing for clean tests of theoretical predictions and simplifying data analysis. However, consistent with the existing literature (Charness and Dufwenberg 2009; Cooper and

\footnotetext{
${ }^{23}$ See Page 212, Game Theory for Applied Economists, 1992 by Princeton University press.
} 
Kühn 2014), we find that restricted signaling has less behavioral impact than free-form communication.

We conclude by discussing three future research ideas that extend on our study. The classification procedure we adopt includes a $2 \times 2$ message space: two $E$-meanings and two $A$ meanings. However, a likely feature of natural language is that attitudes are more finely-grained. For instance, two demanding messages may differ in that one is more demanding than the other. If so, players may be able to take advantage of a common understanding of more subtle distinctions in attitude to achieve coordination. It would be interesting in our setting to implement classification procedures which include more attitude categories. We speculate that doing so would reduce differences between restricted (predetermined) communication with twodimensional meanings and free-form communication.

Our model departs from other intention signaling papers (e.g. Farrell 1987, 1988; Rabin 1991, 1994) principally in the nature of the multi-dimensional language space. We intentionally focused on a pure coordination game so that social preferences do not complicate our analysis. In this environment the use and effect of communication is more readily discerned. Of course, many natural environments of interest cannot be modeled as pure coordination games (e.g. when players' interests are not perfectly aligned), and social preferences do play a ubiquitous role in determining economic choices. An important example is deception when players may benefit from misleading other participants. The role of multi-meaning messages in environments that include the possibility of strategic signaling, and especially when messages can be "deceptive" or "partially deceptive", is an important open question for future investigation.

In relation to restricted language, natural language communication might better enhance group-identity. This might then promote lie-aversion or guilt-aversion, and might also improve one's understanding of the connections between one's decisions and one's outcomes ${ }^{24}$. In our experiment, group-identity likely plays a rather small role given that one-shot simultaneous communication occurs between anonymously paired subjects, with neither feedback nor face-toface interaction. Understanding how multi-meaning natural language enhances group-identity would be particularly useful.

\footnotetext{
${ }^{24}$ See Frey and Bohnet (1995), Valley et al. (1998), Mohlin and Johannesson (2008), Cooper and Kühn (2015 Working Paper).
} 


\section{REFERENCES}

Aumann, R. J. (1974). "Subjectivity and Correlation in Randomized Strategies," Journal of Mathematical Economics 1, 67-96

Aumann, R. J. (1990). “Nash-Equilibria Are Not Self-Enforcing,” Economic Decision Making: Games, Econometrics and Optimisation (J. Gabszewicz, J.-F. Richard, and L. Wolsey, Eds.) Amsterdam: North-Holland. 201-206

Binger B.R., Hoffman, E., Libecap, G.D. (1990) “An Experimentric Study of the Cournot Theory of Firm Behavior," Working paper, University of Arizona

Blume, A., Ortmann, A. (2007) "The Effects of Costless Pre-play Communication:

Experimental Evidence from Games with Pareto-ranked Equilibria,” Journal of Economic Theory, 132: 274-290

Brandts, J., Cooper, D. (2007). "It's What You Say, Not What You Pay: An Experimental Study of Manager-employee Relationships in Overcoming Coordination Failure," Journal of the European Economic Association, 5(6): 1223-1268

Cason, T., Sheremeta, R., Zhang, J. (2012) "Communication and Efficiency in Competitive Coordination Games," Games and Economic Behavior, 76: 26-43

Charness, G. (2000), “Self-Serving Cheap Talk: A Test of Aumann’s Conjecture,” Games and Economic Behavior 33, 177-194

Charness, G., Dufwenberg, M. (2006), “Promises and Partnership,” Econometrica, 74 (6): $1579-1601$

Charness, G., Dufwenberg, M. (2010), "Bare Promises: An Experiment," Economic Letters, 107: $281-283$

Charness, G., Grosskopf, B. (2004), "What Makes Cheap Talk Effective? Experimental Evidence," Economics Letters, 83: 383-389

Chen, Y., Jeon, G., Kim, Y. (2014) “A Day without a Search Engine: An Experimental Study of Online and Offline Searches," Experimental Economics, 17:512-536

Clark, K., Kay, S., Sefton, M. (2001), “When Are Nash Equilibria Self-enforcing? An Experimental Analysis," International Journal of Game Theory, 29:495-515

Cooper, R., Dejong, D.V., Forsythe, R., and Ross, T.W. (1989), "Communication in the Battle of the Sexes Games: Some Experimental Results," RAND Journal of Economics 20(4), $568-587$ 
Cooper, R., Dejong, D.V., Forsythe, R., and Ross, T.W. (1992), “Communication in Coordination Games," Quarterly Journal of Economics, 53: 739-771

Cooper, D., Kagel, J. (2005) “Are Two Heads Better Than One? Team versus Individual Play in Signaling Games," American Economic Review, 95(3): 447-509

Cooper, D. Kühn, K. (2014) "Communication, Renegotiation, and the Scope for Collusion," American Economic Journal: Microeconomics, 6(2): 247-278

Cooper, D. Kühn, K. (2015) “Communication and Cooperation: A Methodological Study," Working Paper

Crawford, V. (1998) "A Survey of Experiments on Communication via Cheap Talk," Journal of Economic Theory, 78: 286 -298

Crawford, V. (2003) "Lying for Strategic Advantage: Rational and Boundedly Rational Misrepresentation of Intentions," American Economic Review, 93: 133-149

Daughety, A., Forsythe, R. (1987) "Regulation and the Formation of Reputations: A Laboratory Analysis," in Public Regulation: New Perspectives on Instituions and Policies, E. Bailey, ed., MIT press

Daughety, A., Forsythe, R. (1987) "The Effects of Industry-wide Price Regulation on Industrial Organization," Journal of Law, Economics, \& Organization, 3(2): 397-434

Dawes, R.M., McTavish, J., Shaklee, H. (1977) "Behavior Communication and Assumptions about Other People's Behavior in a Commons Dilemma Situation," Personality and Social Psychology, 35(1): 1-11

Deck, C., Servátka, M., Tucker (2013) “An Examination of the Effect of Messages on Cooperation under Double-blind and Single-blind Payoff Procedures," Experimental Economics, 16:597- 607

Demichelis, S., Weibull, J.W. (2008) “Language, Meaning, and Games: A Model of Communication, Coordination and Evolution," American Economic Review, 98(4):1292-1311

Duffy, J., Feltovich, N. (2002) “Do Actions Speak Louder Than Words? An Experimental Comparison of Observation and Cheap Talks," Games and Economic Behavior, 39:1-27

Duffy, J., Feltovich, N. (2006) "Words, Deeds, and Lies: Strategic Behavior in Games with Multiple Signals," Review of Economic Studies, 73(3): 669-688

Ellingsen, T., Johannesson, M. (2007) “Anticipated Verbal Feedback Induces Altruistic Behavior," Evolution and Human Behavior, 29(2): 100-105 
Ellingsen, T., Östling, R. (2010) "When Does Communication Improve Coordination,” American Economic Review, 100 (4): 1695-1724

El-Gamal, M., Grether, D. (1995) “Are People Bayesian? Uncovering Behavioral Strategies," Journal of the American Statistical Association 90(432): 1137-1145

Farrell, J. (1987) “Cheap talk, Coordination, and Entry," RAND Journal of Economics, $18: 34-39$

Farrell, J. (1988). “Communication and Nash Equilibrium," Economic Letters, 27: 209-214

Farrell, J. (1993) "Meaning and Credibility in Cheap Talk Games," Games and Economic Behavior, 5: 514-531

Farrell, J., Maskin, E. (1987) “Renegotiation in Repeated Games," Games and Economic Behavior, 1(4): 327-360

Farrell, J., Rabin, M. (1996), “Cheap Talk,” Journal of Economic Perspectives, 10 103-118

Gächter, S., Nosenzo, D., Sefton, M. (2013) "Peer Effects in Pro-social Behavior: Social Norms or Social Preferences?" Journal of the European Economic Association, 11(3): 548-573

Frey B., Bohnet I. (1995), "Institutions Affect Fairness: An Experimental Investigation," Journal of Institutional and Theoretical Economics, 151/2:286-303

Hennig-Schmidt, H., Li, Z.-Y., Yang, C. (2008) "Why People Reject Advantageous Offers Non-monotone Strategies in Ultimatum Bargaining," Journal of Economic behavior and Organization, 65:373-384

Houser, D., Xiao, E. (2011), “Classification of Natural Language Messages Using A Coordination Game," Experimental Economics, 14(1); 1-14

Holm, H.J. (2000) "Gender-Based Focal Points," Games and Economic Behavior 32, 292314

Holt, C., Davis, D. (1990) “The Effects of Non-binding Price Announcements on Postedoffer Markets," Economics Letters, 34 (4): 307-310

Isaac, R.M., Ramey, V., Wlliams, A. (1984) "The Effects of Market Organization on Conspiracies in Restraint of Trade," Journal of Economic Behavior \& Organization, 5(2): 191222

Isaac, R.M., Walker, J. (1988) "Communication and Free Riding Behavior: the Voluntary Contributions Mechanism," Economic Inquiry 26(4): 585-608 
Kimbrough, E., Smith, V., Wilson, B. (2008) "Historical Property Rights, Sociality, and the Emergence of Impersonal Exchange in Long Distance Trade," American Economic Review, 98(3): 1009-39

Lundquist, T., Ellingsen, T., Gribbe, E., Johannesson, M. (2009) "The Aversion to Lying," Journal of Economic Behavior and Organization, 70: 81-92

Marschak, J. (1965) “Economics of Language,” Behavioral Science, 10 (2): 135-140

Mohlin, E., Johannesson, M. (2008) “Communication: Content or Relationship,” Journal of Economic Behavior \& Organization, 65: 409-419

Myers, S., Majluf, N., (1984) “Corporate Financing and Investment Decisions When Firms Have Information that Investors Do Not Have,” Journal of Financial Economics, 13:187-221

Ong, Q., Riyanto, Y., Sheffrin, S., "How Does Voice Matter? Evidence from the Ultimatum Game," Experimental Economics, 15: 604-621

Rabin, M. (1991) "Focal Points in Pre-Game Communication,” Working paper, Department of Economics, UCB. UC Berkeley: Department of Economics, UCB

Rabin, M. (1994) “A Model of Pre-game Communication,” Journal of Economic Theory 63: 370-391

Rubinstein, A. (2000) "Economics and Language: Five Essays," Cambridge University Press

Schelling, T.C. (1960) "The Strategy of Conflict," Cambridge MA: Harvard University Press

Selten, R., Warglien, M. (2007), “The Emergence of Simple Languages in an Experimental Coordination Game," PNAS, 104 (18): 7361-7366

Spence, M. (1973), “Market Signaling,” Quarterly Journal of Economics, 87:355-74

Schotter, A., Sopher, B. (2007) "Advice and Behavior in Intergenerational Ultimatum Games: An Experimental Approach,” Games and Economic Behavior, 58: 365-393

Sutter, M., Strassmair, C. (2009) "Communication, Cooperation and Collusion in Team Tournaments - An Experimental Study," Games and Economic Behavior, 66: 506-525

Valley, K., Moag, J., Bazerman, M. (1998) “A Matter of Trust: Effects of Communication on the Efficiency and Distribution of Outcomes," Journal of Economic Behavior and Organization, 34: 211-238 
Van Huyck, J., Battalio, R., Beil, R. (1990) “Tacit Coordination Games, Strategic Uncertainty, and Coordination Failure," American Economic Review, 80: 234-248

Van Huyck, J., Battalio, R., Beil, R. (1993) “Asset Markets as an Equilibrium Selection Mechanism: Coordination Failure, Game Form Auctions, and Tacit Communication," Games and Economic Behavior, 5: 485-504

Wittgenstein, L. (1957) “The Philosophical Investigations,” The Macmillan Company, New York

Xiao, E. and Houser, D. (2005). "Emotion Expression in Human Punishment Behavior," Proceedings of the National Academy of Sciences of the United States of America, 102(20): 7398-7401

Xiao, E., Houser, D. (2007) "Emotion Expression and Fairness in Economic Exchange," Manuscript, George Mason University 


\section{APPENDIX - PROOFS}

PROOF OF PROPOSITION 1: $\forall \varepsilon_{k} \in \mathcal{E}$, by definition it is necessarily true that $\pi_{i}\left(s_{i}^{\prime}, s_{j}^{\varepsilon_{k}}\right) \leq$ $\pi_{i}\left(s_{i}^{\varepsilon_{k}}, s_{j}^{\varepsilon_{k}}\right), \forall s_{i}^{\prime} \in S_{i}$ and $s_{i}^{\prime} \neq s_{i}^{\varepsilon_{k}}, \forall i, j \in\{1,2\}, j \neq i$. Given $m_{i} \in Q\left(\varepsilon_{k}\right)$ and the assumption of Credibility assumption (Assumption 1), it follows that Player $i$ honors the E-meaning of her message by playing $\varepsilon_{k}$.

PROOF OF PROPOSITION 2: Let $r_{l y}^{j}, r_{l w}^{j}, r_{k y}^{j}, r_{k w}^{j}$ be player is belief about the likelihood they will receive messages $m_{j} \in Q\left(\varepsilon_{l}\right) \cap D\left(\mathcal{A}_{y}\right), m_{j} \in Q\left(\varepsilon_{l}\right) \cap D\left(\mathcal{A}_{w}\right), m_{j} \in Q\left(\varepsilon_{k}\right) \cap$ $D\left(\mathcal{A}_{y}\right), m_{j} \in Q\left(\mathcal{E}_{k}\right) \cap D\left(\mathcal{A}_{w}\right)$ respectively. Given the completeness, we have $r_{l y}^{j}+r_{l w}^{j}+r_{k y}^{j}+$ $r_{k w}^{j}=1$. Suppose $\mathcal{A}_{w}$ indicates stronger desire to have the message followed than $\mathcal{A}_{y}$. For a pure coordination game with equal payoff for two pure-strategy, $\pi_{i}\left(\varepsilon_{l}\right)=\pi_{j}\left(\varepsilon_{l}\right)=\pi_{i}\left(\varepsilon_{k}\right)=$ $\pi_{j}\left(\varepsilon_{k}\right)>\pi_{i}\left(\varepsilon_{m}\right)=\pi_{j}\left(\varepsilon_{m}\right)$. Suppose $\pi_{i}\left(\varepsilon_{l}\right)=X$ and $\pi_{i}\left(\varepsilon_{m}\right)=Y$, the expected payoff functions for four messaging strategies can be simplified as below:

$E\left[\Pi_{i}\left(m_{i} \in Q\left(\varepsilon_{l}\right) \cap D\left(\mathcal{A}_{w}\right), \varphi_{i}\left(m_{i}, \cdot\right)\right)\right]=\pi_{i}\left(\varepsilon_{l}\right) \times\left(r_{l y}^{j}+r_{l w}^{j}+r_{k y}^{j}\right)+\pi_{i}\left(\varepsilon_{m}\right) \times r_{k w}^{j}=(X-Y)\left(r_{l y}^{j}+\right.$ $\left.r_{l w}^{j}+r_{k y}^{j}\right)+Y=(X-Y)\left(1-r_{k w}^{j}\right)+Y$

$E\left[\Pi_{i}\left(m_{i} \in Q\left(\varepsilon_{l}\right) \cap D\left(\mathcal{A}_{y}\right), \varphi_{i}\left(m_{i}, \cdot\right)\right)\right]=\pi_{i}\left(\varepsilon_{l}\right) \times\left(r_{l y}^{j}+r_{l w}^{j}\right)+\pi_{i}\left(\varepsilon_{k}\right) \times r_{k w}^{j}+\pi_{i}\left(\varepsilon_{m}\right) \times$ $r_{k y}^{j}=\pi_{i}\left(\varepsilon_{l}\right) \times\left(r_{l y}^{j}+r_{l w}^{j}+r_{k w}^{j}\right)+\pi_{i}\left(\varepsilon_{m}\right) \times r_{k y}^{j}=(X-Y)\left(r_{l y}^{j}+r_{l w}^{j}+r_{k w}^{j}\right)+Y=(X-Y)\left(1-r_{k y}^{j}\right)+Y$

$E\left[\Pi_{i}\left(m_{i} \in Q\left(\varepsilon_{k}\right) \cap D\left(\mathcal{A}_{w}\right), \varphi_{i}\left(m_{i}, \cdot\right)\right)\right]=\pi_{i}\left(\varepsilon_{k}\right) \times\left(r_{k y}^{j}+r_{k w}^{j}+r_{l y}^{j}\right)+\pi_{i}\left(\varepsilon_{m}\right) \times r_{l w}^{j}=(X-$ $Y)\left(r_{k y}^{j}+r_{k w}^{j}+r_{l y}^{j}\right)+Y=(X-Y)\left(1-r_{l w}^{j}\right)+Y$

$E\left[\Pi_{i}\left(m_{i} \in Q\left(\varepsilon_{k}\right) \cap D\left(\mathcal{A}_{y}\right), \varphi_{i}\left(m_{i}, \cdot\right)\right)\right]=\pi_{i}\left(\varepsilon_{k}\right) \times\left(r_{k y}^{j}+r_{k w}^{j}\right)+\pi_{i}\left(\varepsilon_{l}\right) \times r_{l w}^{j}+\pi_{i}\left(\varepsilon_{m}\right) \times$ $r_{l y}^{j}=\pi_{i}\left(\varepsilon_{k}\right) \times\left(r_{k y}^{j}+r_{k w}^{j}+r_{l w}^{j}\right)+\pi_{i}\left(\varepsilon_{m}\right) \times r_{l y}^{j}=(X-Y)\left(r_{k y}^{j}+r_{k w}^{j}+r_{l w}^{j}\right)+Y=(X-Y)\left(1-r_{l y}^{j}\right)+Y$

Without loss of generality, we assume $r_{l w}^{j}=\min \left\{r_{l y}^{j}, r_{l w}^{j}, r_{k y}^{j}, r_{k w}^{j}\right\}$. Then comparing the four expected utility functions, we have $E\left[\Pi_{i}\left(m_{i} \in Q\left(\varepsilon_{k}\right) \cap D\left(\mathcal{A}_{w}\right), \varphi_{i}\left(m_{i}, \cdot\right)\right)\right]$ has the highest expected payoff, thus $m_{i} \in Q\left(\varepsilon_{k}\right) \cap D\left(\mathcal{A}_{w}\right)$ is the best ex-ante messaging strategy. 